\title{
Magnetic Nanoparticles: A Subject for Both Fundamental Research and Applications
}

\author{
S. Bedanta, ${ }^{1}$ A. Barman, ${ }^{2}$ W. Kleemann, ${ }^{3}$ O. Petracic, ${ }^{4}$ and T. Seki ${ }^{5}$ \\ ${ }^{1}$ School of Physical Sciences, National Institute of Science Education and Research (NISER), IOP Campus, \\ Bhubaneswar 751005, India \\ ${ }^{2}$ Department of Condensed Matter Physics and Material Sciences, S. N. Bose National Centre for Basic Sciences, \\ Block-JD, Sector-III, Salt Lake City, Kolkata 700098, India \\ ${ }^{3}$ Department of Physics, University Duisburg-Essen, 47057 Duisburg, Germany \\ ${ }^{4}$ Jülich Centre for Neutron Science JCNS and Peter Grünberg Institute PGI, JARA-FIT, Forschungszentrum Jülich GmbH, \\ 52425 Jülich, Germany \\ ${ }^{5}$ Institute for Materials Research, Tohoku University, 2-1-1 Katahira, Aoba-ku, Sendai 980-8577, Japan
}

Correspondence should be addressed to S. Bedanta; sbedanta@niser.ac.in

Received 16 July 2013; Accepted 19 October 2013

Academic Editor: Gaurav Mago

Copyright ( $\odot 2013$ S. Bedanta et al. This is an open access article distributed under the Creative Commons Attribution License, which permits unrestricted use, distribution, and reproduction in any medium, provided the original work is properly cited.

Single domain magnetic nanoparticles (MNPs) have been a vivid subject of intense research for the last fifty years. Preparation of magnetic nanoparticles and nanostructures has been achieved by both bottom-up and top-down approaches. Single domain MNPs show Néel-Brown-like relaxation. The Stoner-Wohlfarth model describes the angular dependence of the switching of the magnetization of a single domain particle in applied magnetic fields. By varying the spacing between the particles, the interparticle interactions can be tuned. This leads to various supermagnetic states such as superparamagnetism, superspin glass, and superferromagnetism. Recently, the study of the magnetization dynamics of such single domain MNPs has attracted particular attention, and observations of various collective spin wave modes in patterned nanomagnet arrays have opened new avenues for on-chip microwave communications. MNPs have the potential for various other applications such as future recording media and in medicine. We will discuss the various aspects involved in the research on MNPs.

\section{Introduction}

Modern technologies aided the invention of various new magnetic materials, synthetic structures, micro- and nanostructures, and metamaterials. Magnetism has come a long way and found applications in a range of multidisciplinary fields in present and future nanotechnologies like nonvolatile magnetic memory [1], magnetic storage media [2], magnetic recording heads [3], magnetic resonance imaging [4], and in biomedicine and health science [5]. Emerging technologies such as spin logic [6,7], spin torque nano-oscillators (STNOs) [8], and magnonic crystals [9] have also become very active.

The new technologies demand the invention of new material properties, which requires structuring of known materials in all three dimensions at various length scales and exploiting dynamical magnetic properties over various timescales. For various applications, exploration of a variety of new phenomena is required, and this ranges from slower processes such as domain wall and magnetic vortex dynamics to faster processes such as spin wave propagation and localization, ultrafast demagnetization, and relaxation. This introduces magnetic structures at various length scales such as nanodots, microdisks, magnetic nanowires, and nanostripes. Here, we shall review the experimental and numerical studies of properties of magnetic nanoparticles (MNPs) together with their leading preparation techniques.

Research on MNPs has been a vivid research subject over the last few decades not only for technological reasons, but also from the fundamental research point of view. The field of MNPs has been an interdisciplinary subject, where researchers from physics, chemistry, and biology put equal interest in synthesizing, understanding, and moving 
forward for various applications. In the last decade, thorough investigations have been made in the field of nanosized magnetic particles because of their potential for applications such as data storage, memory, magnonic crystals, permanent magnets, in biology, for example, improving the quality of magnetic resonance imaging (MRI), hyperthermic treatment for malignant cells, site-specific drug delivery, and manipulating cell membranes [10, 11]. Frenkel and Dorfman [12] first predicted that a particle of a FM material is expected to consist of a single magnetic domain below a critical size. Kittel [13] made rough estimates of critical particle sizes. An approximate radius of $10-1000 \mathrm{~nm}$ is found for a spherical MNP of a FM material. The magnitude of the magnetic moment $m$ of a particle is proportional to its volume. Such monodomain FM particles can be viewed as a large magnetic unit, each having a magnetic moment of thousands of $\mu_{B}$. Therefore, these single domain magnetic nanoparticles are called "superspins" or "macrospins." Usually, an ellipsoidal shape of the particles is assumed, where the magnetic moments have the tendency to align along the longest axis, which defines the direction of lowest "shape" anisotropy energy [14].

The critical radius $r_{c}$ below which a particle acts as a single domain particle is given by [15]

$$
r_{c} \approx 9 \frac{\left(A K_{u}\right)^{1 / 2}}{\mu_{0} M_{s}^{2}}
$$

where $A$ is the exchange, $K_{u}$ is the uniaxial anisotropy constant, $\mu_{0}$ is the vacuum permeability, and $M_{s}$ is the saturation magnetization. Typical values for $r_{c}$ are about $15 \mathrm{~nm}$ for $\mathrm{Fe}, 35 \mathrm{~nm}$ for $\mathrm{Co}$, and $30 \mathrm{~nm}$ for $\gamma-\mathrm{Fe}_{2} \mathrm{O}_{3}$, while for $\mathrm{SmCo}_{5}$ it is as large as $750 \mathrm{~nm}$ [16]. Depending on size and material, the magnetic moments of single domain particles can be $10^{3}-10^{5} \mu_{B}[17]$.

There are various models for the magnetization reversal of single domain particles. A model for the coherent rotation of the magnetization was developed by Stoner and Wohlfarth [18]. They assumed noninteracting particles with uniaxial anisotropy in which the spins are parallel and rotate at unison.

In this paper, we highlight the state-of-the-art preparation techniques for MNPs, the magnetic states observed in ensembles of MNPs, and also some future applications of MNPs. In the beginning, we go through both bottom-up and top-down approaches for preparing magnetic nanoparticles and nanostructures. We also briefly discuss nanoparticle superlattices or supracrystals and templated self-organization of nanoparticles. Then, we review some of the magnetic ground states such as superparamagnetism, superspin glass, surface spin glass, and superferromagnetism in ensembles of MNPs. Further, we will discuss the recent progress in femto- and picosecond magnetization dynamics in nanomagnets, particularly the ultrafast demagnetization, relaxation, precession of magnetization, and damping in single and arrays of nanomagnets. Later, the applications of magnetic nanoparticles in various fields, particularly in biology, will be highlighted. Finally, we discuss several major issues and challenges in this field of research.

\section{Synthesis of Magnetic Nanoparticles}

There are basically two types of approaches: (i) bottomup and (ii) top-down approaches to synthesize magnetic nanoparticles and nanostructures.

There are several important issues of nanoparticle synthesis [19] such as (i) obtaining a monodisperse particle size distribution; (ii) control of the particle size in a reproducible manner; (iii) obtaining materials with satisfactory high crystallinity and the desired crystal structure; (iv) control over the shape of nanoparticles; (v) stability of the nanoparticles over long time.

Synthesis of MNPs by chemical methods has been widely used in the last few decades because it is one of the cheapest ways of producing large quantities of the desired MNPs. In the chemical method, the particle size can be well controlled in the range from a few nanometers to micrometers. In chemical synthesis, a short burst of nucleation followed by slow controlled growth is critical to produce monodisperse particles [19].

Figure 1 shows the representative procedure for the preparation of MNPs by chemical synthesis. In the first part, the rapid injection of the reagents, often organometallic compounds, into hot surfactant solution induces the simultaneous formation of nuclei [20]. In the second part, reagents are mixed at low temperature and the resulting reaction mixtures are slowly heated in a controlled manner to generate nuclei. The particle growth occurs by subsequent addition of reactive species. The particle size can also be increased by aging at high temperature by Ostwald ripening, during which smaller nanoparticles dissolve and deposit at the bigger ones [19]. In chemical synthesis, the particle size can be controlled by systematically adjusting the various reaction parameters such as time, temperature, and concentration of reagents and stabilizing surfactants. Also during this process, organic reagents can be added, which will form a shell around the magnetic core, an option to avoid any agglomeration of MNPs.

Another important technique to synthesize MNPs is the microemulsion approach. In this technique, two immiscible liquids form a thermodynamic stable isotropic dispersion denominated microemulsion, where the microdomain of one or both liquids is stabilized by an interfacial film of surfactant molecules [21]. Various magnetic nanoparticles have been prepared by the microemulsion technique, some bodies of literature about which are given by references of Lu et al. [21]. Details of various approaches of chemical synthesis of MNPs are described in various recent articles [19, 21-26].

Sun et al. [27] synthesized FePt nanoparticles using a chemical method. FePt is a highly interesting alloy, since $L 1_{0}$-ordering of $\mathrm{FePt}$ leads to the large uniaxial magnetic anisotropy $\left(K_{u} \sim 7 \times 10^{6} \mathrm{~J} / \mathrm{m}^{3}\right)$, which is comparable to the values for rare earth-based permanent magnets. They successfully prepared monodisperse FePt nanoparticles via a reduction process. The MNPs were regularly aligned on the substrate. However, the nanoparticles still included the disordered phase: that is, the $\mathrm{L1}_{0}$-ordering was not complete because of the limited annealing temperature. In addition, the improvement of hard magnetic properties is indispensable 


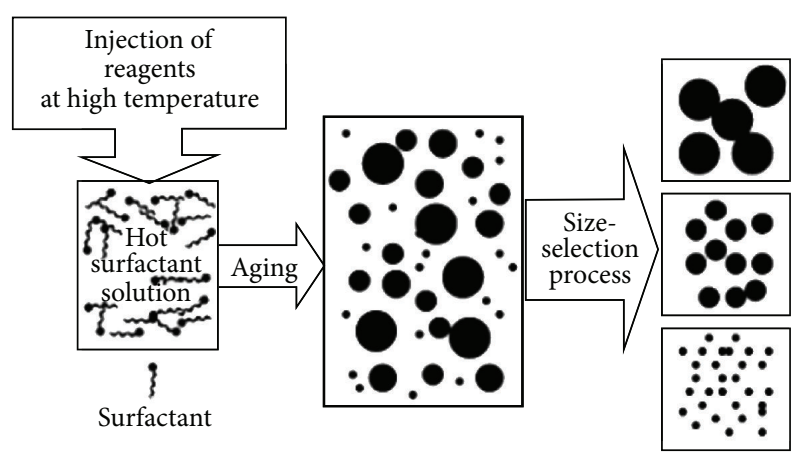

FIGURE 1: The procedure for synthesis of monodisperse nanoparticles by injecting reagents into the hot surfactant solution followed by aging and size-selection process. Reproduced with permission from Hyeon [19] @ 2003, Royal Society of Chemistry.

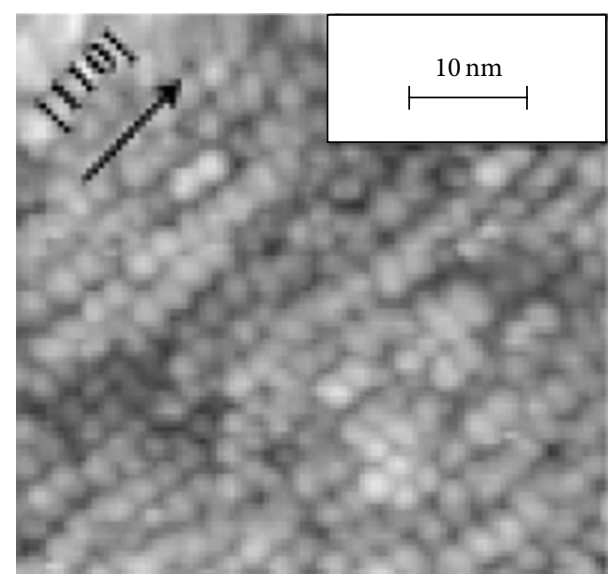

FIGURE 2: Scanning tunneling microscope image of Fe particles deposited on top of an insulating $\mathrm{MgO}$ layer. Reproduced with permission from Ernult et al. [34] (c) 2005, AIP Publishing LLC.

for the applications. Following the above report by Sun et al. [27], many experiments on the preparation of $L 1_{0}-\mathrm{FePt}$ nanoparticles have been carried out using similar solution processes [28-30]. Nanoparticles consisting of exchangecoupled nanocomposites were also fabricated by Zeng et al. [31]. A different chemical method was reported using nanospheres. Self-assembled nanospheres are utilized as a template for the preparation of nanoparticle arrays $[32,33]$. Albrecht et al. [32] formed a topographic pattern of the magnetic multilayer on spherical nanoparticles, where the nanostructures were both monodisperse and magnetically isolated. They found an unexpected switching behavior induced by their spherical shape.

The use of film growth via vapor deposition techniques is another method for self-assembly of nanoparticles. The Volmer-Weber (V-W) mode is a film growth giving an islandlike morphology. This growth mode is achieved by selecting substrate material, layer thickness, and growth temperature adequately. Ernult et al. prepared self-aligned Fe nanoparticles on $\mathrm{MgO}$ employing a molecular beam epitaxy apparatus [34]. Figure 2 shows a typical scanning tunneling microscope image of Fe particles deposited on top of an $\mathrm{MgO}$ layer. They also observed a transition from a three-dimensional V-W type growth mode to a two-dimensional growth, when the layer thickness was increased. Such metallic nanoparticles on an insulating layer become an important component as a structure of electrode/insulating layer/nanoparticles. This allows the injection of an electrical current into the nanoparticles through the insulating layer, playing a major role in devices such as single electron transistors. Black et al. [35] reported the transport measurement performed in selfassembled Co particles. In the case of magnetic nanoparticles, spin-dependent single electron tunneling is expected [36], and Yakushiji et al. [37] found an enhancement of spin lifetime in MNPs.

A particulate film also provides us with an interesting change in the magnetization reversal behavior from the incoherent reversal mode such as domain wall motion to the coherent rotation mode. This change sometimes enhances hard magnetic properties such as coercivity $\left(H_{c}\right)$. Shima et al. [38] deposited $\mathrm{FePt}$ on $\mathrm{MgO}$ at high substrate temperature. When the FePt layer thickness was reduced from $100 \mathrm{~nm}$ to $10 \mathrm{~nm}$, the film morphology was drastically changed from a continuous state to the particulate one as shown in Figure 3. The formation of the particulate film significantly enhanced $H_{c}$ of FePt. They also achieved the huge $H_{c}=105 \mathrm{kOe}$ at $4.2 \mathrm{~K}$ for the FePt particulate film [39]. Okamoto et al. [40] reported that the morphology change with the FePt thickness led to a change in the magnetization reversal process. Further granular films, in which the MNPs are embedded into a nonmagnetic matrix, can isolate the MNPs, which are suitable for next-generation ultrahigh density magnetic recording media.

There are several other methods to prepare MNPs via bottom-up approach such as electrochemical reactions [41], sol-gel processing [42], plasma or flame spraying synthesis [43], chemical vapor deposition [44], atomic or molecular condensation [20], sputtering and thermal evaporation [45], and bio-assisted synthesis [11]. The top-down approach via various types of lithography will be discussed in the next section of this paper.

\section{Magnetic Nanostructures by Lithography}

In contrast to the self-assembly of the bottom-up techniques, the top-down techniques employ the microfabrication processes such as lithographical patterning, liftoff, ion milling, or wet etching. The top-down techniques have advantages that we can accurately control the size and the shape of nanostructures, and it is easy to align the nanostructures as desired. However, high cost and slow manufacturing of the top-down techniques are drawbacks for the mass production. In addition, the size limitation of the nanostructure depends on the kind of lithography. If one uses an electron beam (EB) as a writer, that is, EB lithography, the size can be reduced down to a few tens of nanometer although it takes long time to make patterns. Photolithography saves the time for patterning owing to the large area exposure, while the 

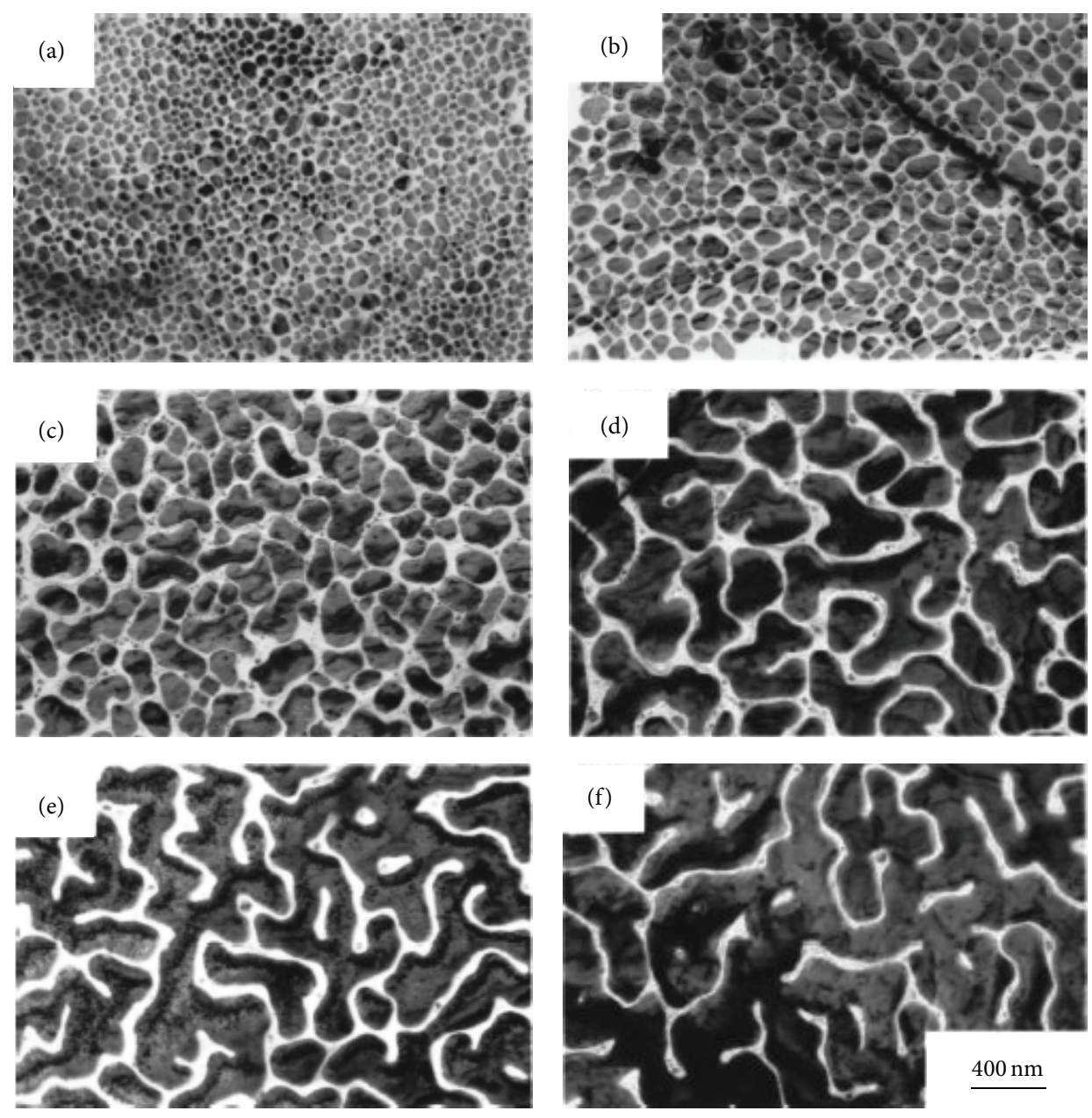

FIgURE 3: Transmission electron microscope images for FePt thin films with thicknesses of (a) $10 \mathrm{~nm}$, (b) $15 \mathrm{~nm}$, (c) $20 \mathrm{~nm}$, (d) $45 \mathrm{~nm}$, (e) $50 \mathrm{~nm}$, and (f) $60 \mathrm{~nm}$. Reproduced with permission from Shima et al. [38] @ 2002, AIP Publishing LLC.

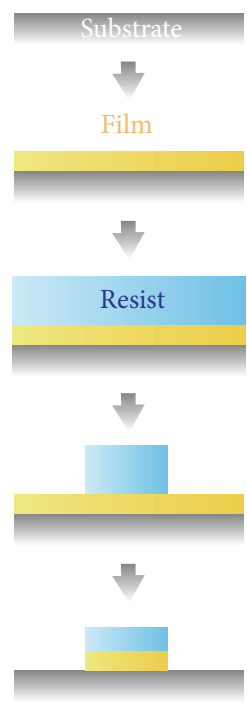

(a) Etching (milling)

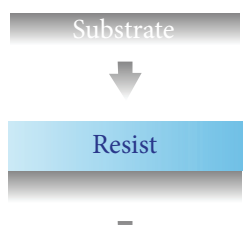

5

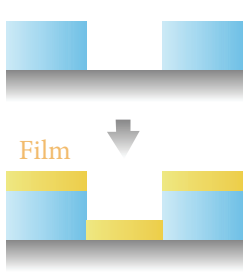

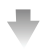

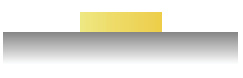

(b) Liftoff
FIGURE 4: Schematic illustrations of the microfabrication processes (a) etching (milling) and (b) liftoff. minimum size is mainly determined by the wavelength of the light source.

Figure 4 illustrates the steps for microfabrication processes using (a) etching or milling, and (b) liftoff. In the case of the etching process, a film is first deposited on a substrate. Then, a resist for lithographical patterning is spincoated onto the film and a desired pattern of the resist is formed. Finally, the film is etched through the resist mask, and the desired pattern is obtained. Contrastingly, a resist is first spin-coated onto a substrate in the case of the liftoff process. After patterning the resist, a film is deposited on the substrate with the patterned resist. The thin film deposited on the region without resist finally remains after lifting the resist off from the substrate. Both methods have advantages and disadvantages. For example, although the lift-off process is free from etching damage, the substrate surface is contaminated by the resist that can be suppressed in the etching process. There are many reports on the magnetic properties for nanostructured elements of $\mathrm{NiFe}$, $\mathrm{Fe}, \mathrm{Co}$, and $\mathrm{Co} / \mathrm{Pt}[47-50]$. Such nanostructures showed the characteristic magnetization reversal behavior and magnetic domain structures. For example, Shinjo et al. [51] reported 

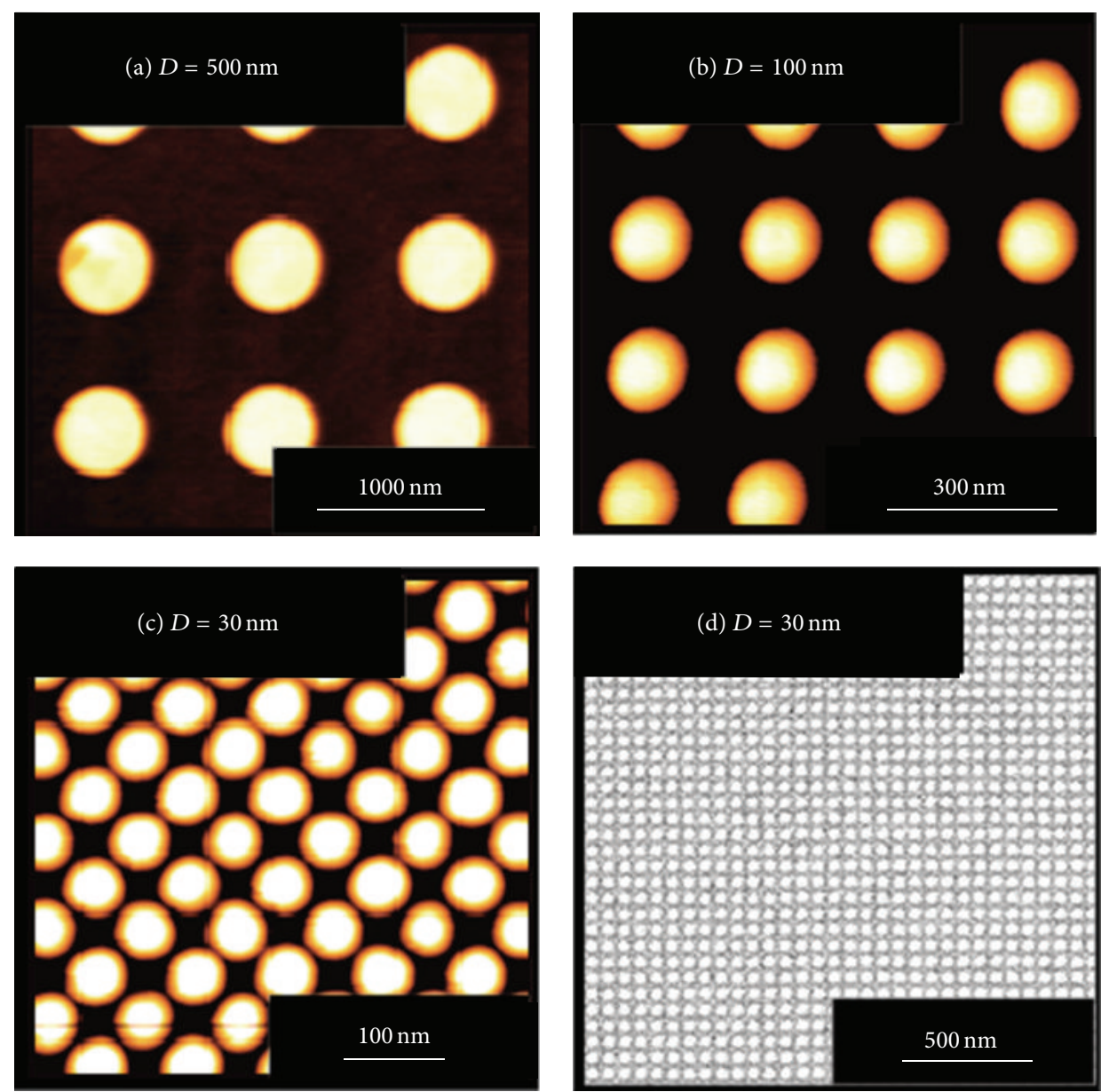

Figure 5: Atomic force microscope images for the $L 1_{0}$-FePt circular dots with diameters (D) of (a) $500 \mathrm{~nm}$, (b) $100 \mathrm{~nm}$, and (c) $30 \mathrm{~nm}$ and (d) a scanning electron microscope image for $D=30 \mathrm{~nm}$. Reproduced with permission from Seki et al. [46] @ 2011, IOP Publishing.

the first observation of magnetic vortex core in NiFe disks prepared using the top-down technique. The formation of the magnetic vortex is attributable to the balance between magnetic exchange energy and magnetostatic energy in a confined magnetic disk. The top-down techniques are also used to fabricate magnetic nanopillars and nanowires, which are key elements for future spintronic devices such as magnetic random access memories [52] and racetrack memories [53], respectively.

As well as the bottom-up technique, one can control the magnetization reversal mode in a magnetic nanostructure fabricated by the top-down technique. Figure 5 displays the atomic force microscope images and the scanning electron microscope image for $L 1_{0}-\mathrm{FePt}$ circular dots with various diameters, which were fabricated through the use of EB lithography and Ar ion milling [46]. These images support that $\mathrm{FePt}$ was patterned into well-defined circular shapes even in the case of the diameter of $30 \mathrm{~nm}$. As the dot diameter was reduced from $1 \mu \mathrm{m}$ to $30 \mathrm{~nm}$, the magnetization reversal mode was changed as in the case of $L 1_{0}-\mathrm{FePt}$ nanoparticles prepared by the bottom-up technique. Figure 6 shows the magnetic field angular dependence of normalized $H_{c}$ for the perpendicularly magnetized $L 1_{0}$ - FePt dots with various dot diameters, where $\theta$ is the polar angle of the magnetic field and $\theta=0^{\circ}$ and $90^{\circ}$ correspond to the normal and parallel directions to the plane of the device. $H_{c}$ was normalized by the value of $H_{c}$ at $\theta=0^{\circ}$. The magnetization reversal for the dots with a large diameter is governed by domain wall motion, where the angular dependence follows the relationship of $1 / \cos \theta$. On the other hand, the $\theta$ dependence of normalized $H_{c}$ deviates from the tendency of $1 / \cos \theta$ with decreasing dot diameter, and the local minimum of $H_{c}$ appears around $\theta=45^{\circ}$. This implies that the magnetization reversal for dots with a small diameter occurs through the incoherent magnetization rotation. A detailed analysis of magnetization reversal was also carried out for a single $\mathrm{FePt}$ dot using the anomalous Hall effect [54]. In addition, the nucleation phenomenon of the reversed magnetic domains was discussed using microfabricated FePt dots [55]. Consequently, nanostructures fabricated by the top-down technique are useful for doing systematic investigations of the magnetization reversal behavior and magnetic domain 


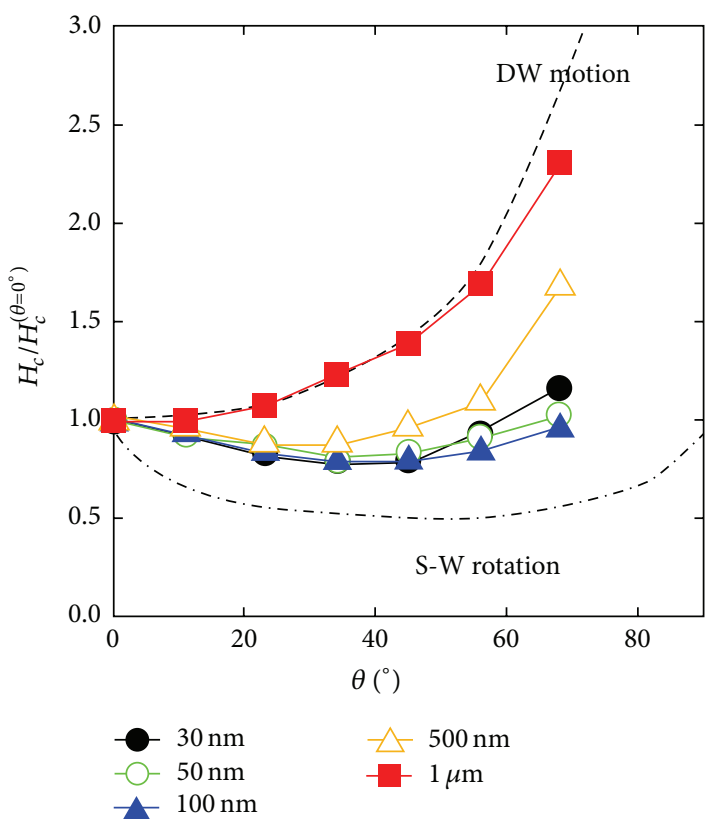

FIGURE 6: Magnetic field angular dependence of normalized coercivity $\left(H_{c}\right)$ for $L 1_{0}$-FePt dots with diameters of $1 \mu \mathrm{m}$ (solid squares), $500 \mathrm{~nm}$ (open triangles), $100 \mathrm{~nm}$ (solid triangles), $50 \mathrm{~nm}$ (open circles), and $30 \mathrm{~nm}$ (solid circles). The dashed line denotes $1 / \cos \theta$, whereas the dashed-dotted line denotes the Stoner-Wohlfarth-type coherent rotation.

structures at the nanometer scale owing to their well-defined geometries.

\section{Nanoparticle Superlattices or Supracrystals}

A particularly exciting novel approach in NP research is to fabricate and to study self-organized assemblies of MNPs [56-58]. They constitute an analogy to conventional crystalline materials because atoms are replaced by MNPs and spins are replaced by superspins. Instead of an atomic crystal, one rather speaks of so-called MNP "supracrystals" or "superlattices" $[58,59]$. Such systems thus represent a new class of materials, where their properties are determined, on the one hand, by the properties of the individual MNPs as their building blocks and, on the other hand, by the mutual interactions. Therefore, interesting behavior and novel applications can be expected from the controlled tuning of individual and collective properties of the superlattices.

NP superlattices can be fabricated by various techniques. Basically one has to distinguish four different classes of methods, namely, as follows.

(i) Physical vapor deposition methods: they include, for example, thermal vapor deposition or sputtering. In this case, the MNPs of magnetic metals such as $\mathrm{Co}_{80} \mathrm{Fe}_{20}$ are formed spontaneously during deposition by nonwetting Volmer-Weber-type growth onto a suitable substrate material like $\mathrm{SiO}_{2}$ and $\mathrm{Al}_{2} \mathrm{O}_{3}$. The advantage is a completely "organics-free" procedure;

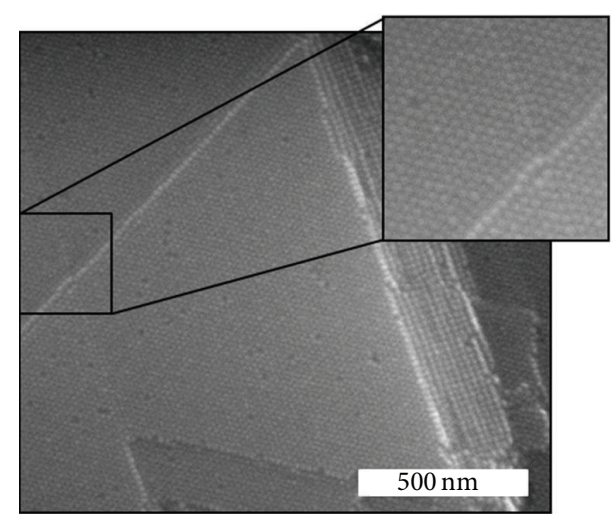

FIGURE 7: Scanning electron microscopy image of a 3-dimensional assembly of iron oxide nanoparticles with $20 \mathrm{~nm}$ diameter on a $\mathrm{Si}$ substrate [62].

however, the order of self-organization of these discontinuous metal-insulator multilayers (DMIMs) is usually relatively weak and the shape of the MNPs is not well controllable $[60,61]$.

(ii) MNPs from the gas phase: here, MNPs are formed, for example, by nucleation and growth from a supersaturated vapor. In a subsequent step, the MNPs are "landed" onto a substrate [63, 64]. Advantages are the ability to form spherical MNPs from many different materials with relatively well-controllable diameters. However, also here, the order of self-organization once deposited is relatively poor.

(iii) Chemically prepared MNPs: in this case, the MNPs are synthesized by chemical processes and are dispersed in a solvent. Self-assembly onto a substrate then occurs by involving colloidal ordering processes during evaporation of the solvent $[21,56-58,62$, 65]. In this case, superlattices of excellent quality can be prepared. Figure 7 shows an example of a 3 -dimensional assembly of $20 \mathrm{~nm}$ iron oxide MNPs [62].

This third class of methods, one could call it "colloidal selfassembly techniques", comprises a large number of various approaches. To mention only a few examples there are $[66,67]$ the following.

(i) "Drop-casting," namely, applying a droplet of the MNP dispersion onto a substrate and letting the solvent evaporate [68]. This method often produces very thick 3-dimensional superlattices of relatively high quality. However, the thickness of the MNP film varies significantly over the substrate surface and basically no control of the self-assembly process is possible.

(ii) "Spin-coating" or rotation-coating, which is similar to coating a substrate with photosensitive or electronbeam sensitive resist in nanolithography. This produces films of very good homogeneity. Even submonolayer MNP films are possible by this approach 


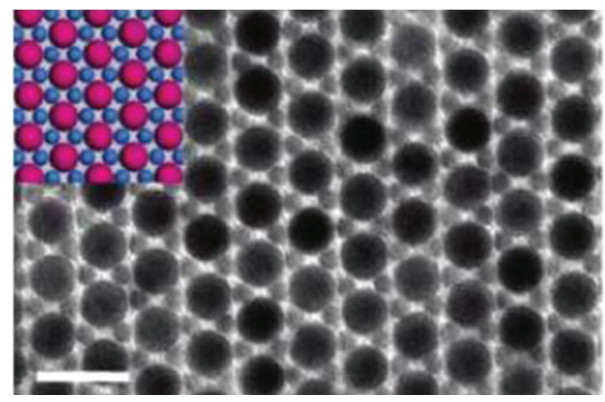

FIGURE 8: TEM image of a binary MNP superlattice composed of 5.6 and $10.5 \mathrm{~nm} \mathrm{Fe}_{3} \mathrm{O}_{4}$ NPs. The inset shows the structural model of the [001] projection of this lattice. The scale bar corresponds to $20 \mathrm{~nm}$. Reproduced with permission from Chen et al. [71] ( ) 2010, American Chemical Society.

[69]. However, one achieves hereby only short-range ordered superlattices, probably due to the extremely quick process, where the MNPs have little time to assemble. The control parameter is-apart from the choice of the solvent as in any other techniquebasically only the spin-speed.

(iii) "Langmuir-Blodgett" technique: in this method a substrate is initially immersed into the MNP dispersion. Using a high-precision motorized stage, the substrate is slowly pulled out of the dispersion at a constant angle and at a constant speed. Both parameters are control parameters of the process [66]. This method is in principle capable of producing highquality monolayer MNP films.

(iv) "Sedimentation": the substrate is placed at the bottom of the container, where initially the MNP dispersion is filled in. The solvent evaporates slowly and the MNPs "sediment" onto the substrate. Control parameters are the evaporation rate, the type of the vessel (e.g., its material), the temperature, and the use of sonication or not.

Further methods are, for example, "Langmuir-Schäfer" and "capillary" techniques [67] and various methods exploiting the self-assembly at a liquid-liquid or liquid-air interface [70]. By such methods, it is possible to fabricate MNP films of excellent order extending over several micrometers and even involving particles of two or three different sizes (see, e.g., Figure 8).

The magnetic properties of such MNP superlattices have been in the focus of many current studies [27, 56, 68, 7276]. In most cases, the collective magnetic behavior of the superlattice-being a consequence of dipolar interactionsis intensely investigated. Although the physics of dipolarly interacting $2 \mathrm{D}$ or $3 \mathrm{D}$ lattices seems to be a "classic" and solved topic in magnetism, the community is far from a consensus. This is due to the huge complexity of the system, where dipolar interactions are long-range and anisotropic and thus lead to both frustrated and highly correlated behavior like superspin glass or superferromagnetism (see Section 7). Therefore, the behavior of such arrays is hard to predict and many parameters like shape of the entire system, anisotropies, order versus disorder, and many more influence the properties significantly.

\section{Superparamagnetism}

Small enough FM particles will be single domain, since the energy cost of domain wall formation outweighs any saving of demagnetizing energy. In these single domain FM particles, the magnetization is often considered to lie parallel or antiparallel to a particular direction called the easy axis. This can be due to different anisotropy contributions, for example, magnetocrystalline, shape, strain, and surface anisotropies [77]. Let us consider an assembly of uniaxial single domain particles, each with an anisotropy energy density $E=K V \sin ^{2} \theta$. For a particle, the energy barrier $\Delta E_{B}=K V$ separates the two energy minima at $\theta=0$ and $\theta=\pi$ corresponding to the magnetization parallel or antiparallel to the easy axis as shown in Figure 9. Néel pointed out [78] that for small enough single domain particles $K V$ may become so small that energy fluctuations can overcome the anisotropy energy and spontaneously reverse the magnetization of a particle from one easy direction to the other, even in the absence of an applied field. In the limit $k_{B} T \gg K V$, the particle can be considered freely fluctuating $\left(k_{B}=\right.$ Boltzmann's constant). A FM nanoparticle is defined as superparamagnetic (SPM), when the energy barrier, $E_{B}$, for a magnetization reversal is comparable to the thermal energy, $k_{B} T$. This behavior has been discussed in the literature also under several other names, including "apparent paramagnetism" [79], "collective paramagnetism" [80], "quasiparamagnetism" [81], and "subdomain behavior" [82]. This is the isotropic SPM limit. The direction of the superspin or macrospin fluctuates with a frequency $f$ or a characteristic relaxation time, $\tau=(2 \pi f)^{-1}$. The Néel-Brown expression $[78,83]$ for the relaxation time is given by

$$
\tau=\tau_{0} \exp \left(\frac{K V}{k_{B} T}\right),
$$

where $\tau_{0} \sim 10^{-10} \mathrm{~s}$ is the inverse angular attempt frequency. The fluctuations thus slow down ( $\tau$ increases) as the sample is cooled to lower temperatures. The system appears static when the SPM relaxation time $\tau$ becomes much longer than the experimental measuring time $\tau_{m}$. Only if $\tau$ is shorter compared to $\tau_{m}$, one may observe an average value of the magnetization. When this SPM relaxation time $\tau$ becomes comparable to $\tau_{m}$, the particle is said to be blocked. The magnetic behavior of the particle is characterized by the so-called "blocking" temperature, $T_{b}$, below which the particle moments appear frozen on the time scale of the measurement, $\tau_{m} \approx \tau$. Inverting (2), one obtains

$$
T_{b} \approx \frac{K V}{k_{B} \ln \left(\tau_{m} / \tau_{0}\right)} .
$$

Equation (3) is valid for individual particles or a system of noninteracting particles with the same size and anisotropy. If the particles are not monodisperse, the distribution of 


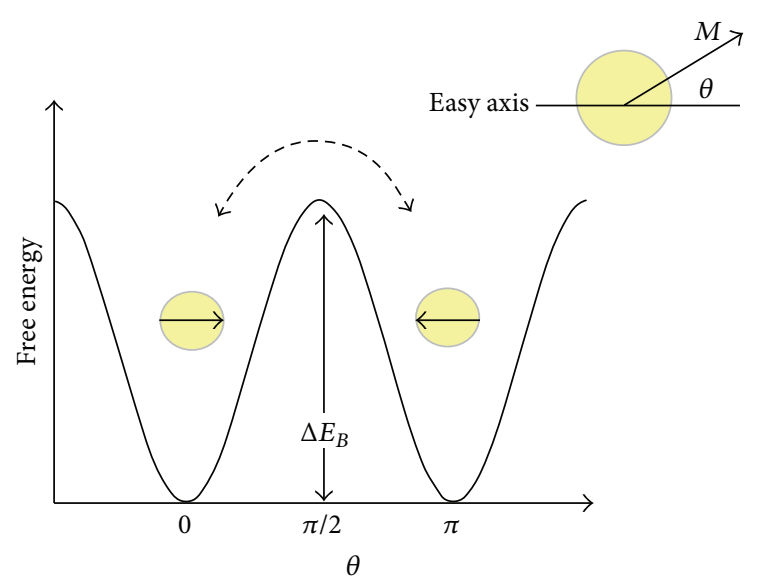

FIgURE 9: Schematic picture of the free energy of a single domain particle with uniaxial anisotropy as a function of magnetization direction. $E_{B}$ is the energy barrier hindering the free rotation of the magnetization and $\theta$ is the angle between the magnetization $\mathbf{M}$ and the easy axis.

particle sizes results in a distribution of blocking temperatures. The experimental measuring time $\tau_{m}$ is different from one measurement technique to another. For example, $\tau_{m}$ is in the range $10^{-12}-10^{-10} \mathrm{~s}$ for inelastic neutron scattering and time-resolved magneto-optical Kerr effect measurement, $10^{-10}-10^{-7} \mathrm{~s}$ for Mössbauer spectroscopy (comparable to the decay time of the nuclear Mössbauer transition), and $10^{-10}$ $10^{-5} \mathrm{~s}$ for $\mu \mathrm{SR}$ (a measurable fraction of muons live for up to $\sim 10 \tau_{\mu}$, where $\tau_{\mu}=2.2 \mu$ s is the average muon lifetime), while ac susceptibility typically probes $10^{-1}-10^{-5} \mathrm{~s}$. Therefore, it should be noted that for a specific sample the blocking temperature is not uniquely defined, but for each applied experimental technique a related blocking temperature must be defined. Brown [84] has shown that $\tau_{0}$ depends on the material parameters (size and anisotropies), field, and even on temperature. From (3), it is clear that $\tau$ depends on $V$ and $T$ so that by varying the volume of the particles or the measurement temperature, $\tau$ can be in the order from $10^{-9} \mathrm{~s}$ to several years (Figure 9).

It should be noted that the SPM blocking phenomena can be observed in ensembles of MNPs with negligible or very weak interparticle interactions. However, interactions can affect the inter-particle magnetic states such as superspin glass and superferromagnetism, which will be discussed in the next two sections.

\section{Superspin Glass and Surface Spin Glass}

Spin glasses (SG) belong to the most prominent disordered systems in solid state magnetism and have thoroughly been investigated for decades [85]. With a few quite general ingredients such as site disorder and frustrated interaction they generate an amazing wealth of properties. They culminate in the definition of the SG order parameter, which sharply contrasts to that of ferro- or antiferromagnets but meaningfully describes the random distribution of frozen spin orientations [86]. An often studied example is the disordered alloy $\mathrm{Cu}_{1-x} \mathrm{Mn}_{x}$ with $x \ll 1$ [87], whose random $\mathrm{Mn}$ spin distribution and oscillating indirect RKKY exchange interaction provides the key ingredients of glassiness.

Analogously to the SG state of dilute spins in bulk materials, a collective glassy magnetic state can also occur in ensembles of single domain NPs in which the interparticle interaction is nonnegligible. Under the condition of a nonvanishing NP magnetization ("superspin"), $\langle S\rangle \neq 0$, being interpreted either by that of a finite-size ferromagnet or by the ground state of a magnetic macromolecule, superspin glass (SSG) states have been considered, for example, in frozen ferrofluids [88] or discontinuous metal-insulator multilayers (DMIMs) [75]. From the beginning, the crucial ingredientsspatial randomness and frustration-were evident when accepting magnetic dipolar interaction to prevail between the superspins. Similar arguments as in classic dipolar glasses [89] were accepted by most researchers with very few exceptions [90].

Figure 10 shows some of the key signatures of a typical DMIM SSG, namely, $\left[\mathrm{Co}_{80} \mathrm{Fe}_{20}\left(t_{n}=0.9 \mathrm{~nm}\right) / \mathrm{Al}_{2} \mathrm{O}_{3}(3 \mathrm{~nm})\right]_{10}$ [91]. The typical low- $T$ shift of the peak temperature $T_{m}$ of the broad glassy susceptibility response under decreasing frequencies $10^{-2} \leq f \leq 10^{2} \mathrm{~Hz}$ is shown in Figure 10(a). The dominating relaxation time $\tau=(2 \pi f)^{-1}$ turns out to obey a critical power law,

$$
\tau=\tau^{*}\left(\frac{T_{m}}{T_{g}}-1\right)^{-z \nu},
$$

with reasonable parameters referring to the glass temperature $T_{g}=61 \mathrm{~K}$, the dynamic critical exponent $z v=10.2$, and the relaxation time of an individual particle moment $\tau^{*}=10^{-8} \mathrm{~s}$, Figure 10(c). An Arrhenius ansatz, Figure 10(b), $\tau=\tau_{0} \exp \left(K V / k_{B} T_{m}\right)$, also seems to fit with a reasonable anisotropy parameter $K V=2.4 \times 10^{-20} \mathrm{~J}$ but yields an unreasonably small value of the inverse angular attempt frequency, $\tau_{0}=10^{-23} \mathrm{~s}$, and can thus be excluded.

Nearly simultaneously with the first report on a SSG [88] another nanoparticular peculiarity was reported by Kodama et al. [92], namely, the surface spin disorder in $\mathrm{NiFe}_{2} \mathrm{O}_{4} \mathrm{NPs}$. A model of the intrananoparticular magnetization involving ferrimagnetically aligned core spins and a spin-glass-like surface layer with a canted spin structure (Figure 11(a)) was proposed on the basis of anomalous magnetic low temperature properties and numerical calculations. Ever since this pioneering and highly cited publication, the subject has been in the centre of attention [93]. Only recently important details of the surface spin dynamics in dilutely dispersed $\mathrm{NiFe}_{2} \mathrm{O}_{4}$ NPs were disclosed by Nadeem et al. [94]. They drew the attention to the core-shell structure, where the ferrimagnetic core is completely blocked at $T_{g} \approx 15 \mathrm{~K}$ and the shell encounters a classic spin glass transition with aging, memory, and dynamic scaling effects.

Disorder and frustration, the classic ingredients of SG formation, are readily available at surfaces of ferrimagnetic (such as $\mathrm{NiFe}_{2} \mathrm{O}_{4}$ ) or antiferromagnetic particles (such as $\mathrm{NiO}$ [95]). Figure 11(b) shows its separate peak-like anomalies of the complex ac susceptibility, $\chi^{\prime}-i \chi^{\prime \prime}$, at the blocking 


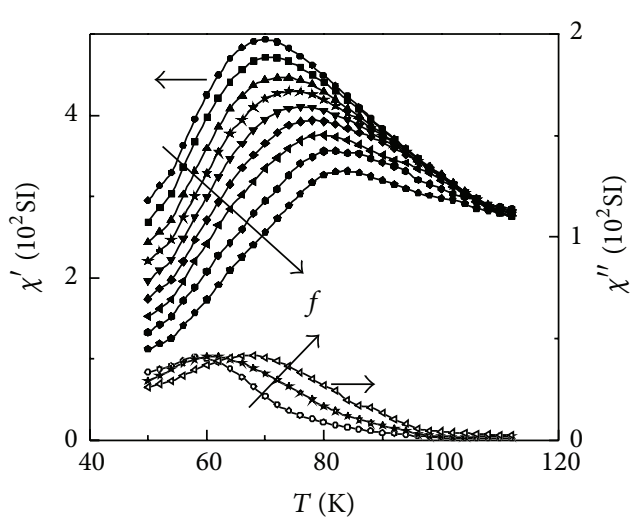

(a)

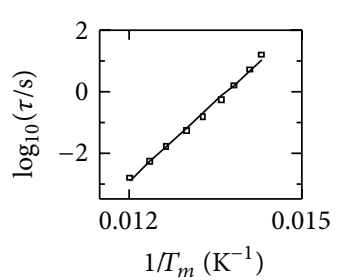

(b)

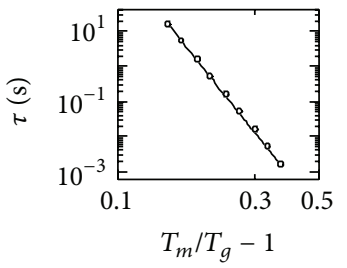

(c)

FIGURE 10: (a) Real and imaginary components, $\chi^{\prime}(T)$ and $\chi^{\prime \prime}(T)$, of the $a c$ susceptibility measured on $\left[\mathrm{Co}_{80} \mathrm{Fe}_{20}\left(t_{n}=0.9 \mathrm{~nm}\right) / \mathrm{Al}_{2} \mathrm{O}_{3}(3 \mathrm{~nm})\right]_{10}$ at frequencies $10^{-2} \leq f \leq 10^{2} \mathrm{~Hz}$. (b) Arrhenius law fit (straight line) to $\log _{10}[\tau / s]$ versus $1 / T_{m}$, where $T_{m}$ corresponds to the peak position of $\chi^{\prime}(T)$. (c) Double logarithmic plot of $\tau$ versus $T_{m} / T_{g}-1$ and best fit to a power law (straight line). Reproduced with permission from Sahoo et al. [91] () 2003, AIP Publishing LLC.

temperature, $T_{B} \approx 85 \mathrm{~K}$ (inset) and at $T_{g}=15.9 \mathrm{~K}$ as extrapolated from (4) with $z \nu=8$ and $\tau_{0}=10^{-12} \mathrm{~s}$. Even the probably oldest magnetic material in history, magnetite, $\mathrm{Fe}_{3} \mathrm{O}_{4}$, has recently disclosed a surface SG transition, whose frequency-dependent peak of the out-of-phase susceptibility $\chi^{\prime \prime}(T)$ has been located at $\approx 35 \mathrm{~K}$ in NPs sized $40 \mathrm{~nm}$ [96].

\section{Superferromagnetism}

In the superparamagnetic (SPM) state of MNPs, no collective interparticle order exists, while the intraparticle spin structure gives rise to individual net magnetic "supermoments" ("superspins" or "macrospins"). However, for increasing particle concentration the magnetic inter-particle interactions become nonnegligible and one may find a crossover from single-particle blocking to collective freezing. As described above for an intermediate strength of magnetic interactions, randomness of particle positions, and sufficiently narrow size distribution, one can observe a SSG state. With further increase of concentration, but prior to physical percolation, the inter-particle interactions become stronger and finally can lead to a kind of FM domain state. FM-like correlations will arise between the "supermoments" of the nanoparticles in addition to those between the atomic moments within the particles. The FM state of nanoparticle ensembles might therefore be called "superferromagnetic" (SFM). Consequently, a SFM domain is defined like a FM domain, the only difference being that the atomic moments is replaced by the supermoments of the individual nanoparticles.

The term "superferromagnetism" was first introduced by Bostanjoglo and Roehkel [97]. Later Mørup observed it when studying microcrystalline goethite, $\mathrm{FeO}(\mathrm{OH})$, by Mössbauer spectroscopy [98]. Afterwards, the same terminology has been used in different magnetic systems [99]. However, a
SFM domain state has scarcely been evidenced up to now in nanoparticle systems. For example, Sankar et al. [100] have studied nonpercolated $\mathrm{Co}-\mathrm{SiO}_{2}$ granular films and evidenced FM-like correlations between the nanoparticles by smallangle neutron scattering. In their case, the observed magnetic correlations were extracted from the ZFC state in zero magnetic field. That is why they attributed the FM correlations to magnetic interactions among the nanoparticles. On different concentrations of nanoparticles, they found that the FM correlations disappear for lower metallic volume fractions, that is, among others; for weaker dipolar interactions.

There has been indication or evidence for the existence of SFM domains observed by various experiments or experimental protocols such as dynamic hysteresis [101, 102], polarized neutron reflectometry [103], Cole-Cole diagrams [104, 105], and aging and memory effects [104]. However, real time imaging of SFM domains was only possible by Kerr microscopy and X-ray photoemission electron microscopy (X-PEEM) [105]. Figures 12(a)-12(f) show LMOKE micrographs following the temporal evolution of the switching process at room temperature after saturating the negative magnetization (dark) and subsequently exposing the sample to a positive supercoercive field of $\mu_{0} H=0.6 \mathrm{mT}$. The first stripe-like domains with reversed magnetization (light) appear at time $t \approx 2 \mathrm{~s}$, as seen in Figure 12(a). In the next few seconds, they are observed to expand simultaneously sideways and along the easy (=field) direction, while further domains nucleate at other sample regions. These sideways sliding $[102,103]$ and nucleation processes continue under the same constant field, until all of the downmagnetization is reversed after 9 seconds. Systematic investigations have shown that the domain nucleation rate and the velocity of subsequent viscous slide motion of the walls can accurately be controlled by the magnitude of the external field [105]. 


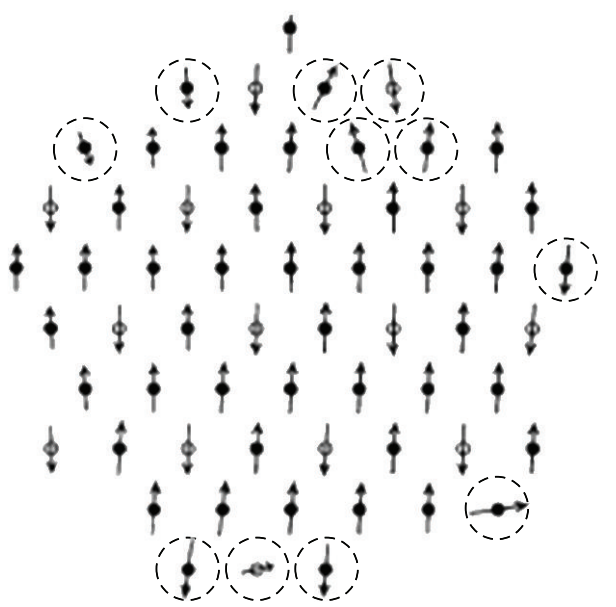

(a)

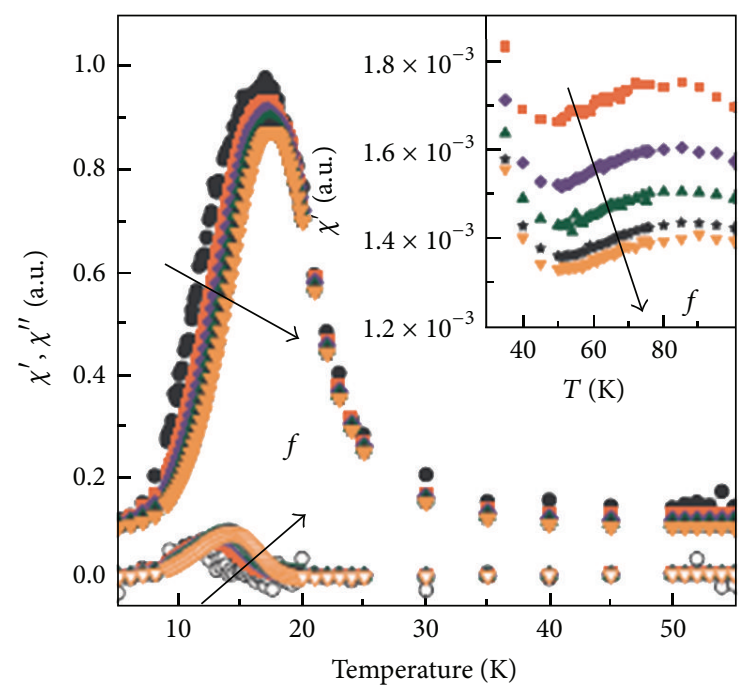

(b)

FIGURE 11: (a) Calculated spin configuration at $H=0$ for a cross-section of a $\mathrm{NiFe}_{2} \mathrm{O}_{4}$ particle with diameter $2.5 \mathrm{~nm}$. Circles indicate extremely canted orientations. Reproduced with permission from Kodama et al. [92] ( ) 1996, APS. (b) Temperature dependence of $\chi^{\prime}$ and $\chi^{\prime \prime}$ (solid and open symbols, resp.) of $\mathrm{NiO}$ particles with mean diameter $6.5 \mathrm{~nm}$ under an $a c$ field of $H_{0}=10 \mathrm{Oe}$, measured at frequencies $10 \mathrm{~Hz} \leq f \leq$ $10 \mathrm{kHz}$ with $\chi^{\prime}$ peaking at $T_{g}=15.9 \mathrm{~K}$ as $f \rightarrow 0$. The inset shows the high temperature region with $\chi^{\prime}$ peaking at $T_{B} \approx 85 \mathrm{~K}$. Reproduced with permission from Winkler et al. [95] () 2008, IOP Publishing.

\section{Magnetic Core-Shell Nanoparticles}

Core-shell magnetic nanoparticles have gained particular attention because of their physical and chemical properties that are strongly dependent on the structure of the core, shell, and interface. It is now known that the values of magnetic anisotropies in small particle exceed the corresponding bulk magnetocrystalline ones by orders of magnitude [106]. Another interest in magnetic core-shell nanoparticles arises because of the possibility to tune the surface strain anisotropy on the magnetic core through core/shell interface microstructure manipulation.

The subject of magnetic core-shell nanoparticles can be divided into three parts: (i) a magnetic core with nonmagnetic shell, (ii) a ferromagnetic core with ferromagnetic shell, and (iii) a ferromagnetic (FM) core with antiferromagnetic (AFM) shell or vice versa. So far, it has been successfully shown in recent years that a nonmagnetic coating can be used for magnetic core stabilization and surface functionalization for applications, for example, in biology $[107,108]$.

An AFM shell coated over a FM core leads to the so-called exchange bias effect. This effect is basically an interfacial undercompensation of spins, which may induce unidirectional anisotropy and is noticed as a shift of the hysteresis loop along the field axis depending on the history of the direction of the cooling field $H_{F C}$. In the AFM/FM coreshell structure, the Curie temperature, $T_{c}$, of the FM has to be higher than the Néel temperature $\left(T_{N}\right)$ and the system has to be cooled from a starting temperature in between in the presence of an applied field $H_{F C}$. It has been shown that exchange bias in core-shell magnetic particles provides a path for the improvement in the thermal stability of the core [109]. EB has been observed in nanoparticles for a variety of materials and morphologies, which can be divided in three categories [110]:

(i) single phase ferromagnetic or antiferromagnetic oxides,

(ii) NPs deposited in AFM matrices,

(iii) NPs with core-shell structure.

It has also been shown that EB-like hysteresis shifts can be observed in FM nanoparticles, in which the surface behaves like a spin glass, which is formed due to finite-size and surface effects [111, 112] (see also Section 6). Exchange bias in coreshell NPs has been found in many types of systems, for example, in $\mathrm{Co} / \mathrm{CoO}[109,113], \mathrm{NiCo} / \mathrm{NiCoO}[114], \mathrm{Co} / \mathrm{MnO}$ [115], $\mathrm{Co}_{80} \mathrm{Ni}_{20}$ /oxide [116], $\mathrm{CoPt} / \mathrm{CoO}[117,118]$, and so forth.

Exchange bias has been reported in unconventional AFM core with ferromagnetic shell, for example, in nanoparticles with $\mathrm{MnO}$ (core) $/ \mathrm{Mn}_{3} \mathrm{O}_{4}$ (shell) [119]. Similar unconventional systems are ferrimagnetic $\mathrm{CoFe}_{2} \mathrm{O}_{4}$ (core)/antiferromagnetic $\mathrm{Mn}$ (shell), $\mathrm{Fe}_{3} \mathrm{O}_{4} /$ Co nanocables [120], and so forth. For details of such exchange bias in core-shell magnetic nanoparticles, readers are referred to references $[109,121]$.

\section{Applications of Magnetic Nanoparticles}

The unique chance to control coercivity in magnetic nanomaterials has led to a number of significant technological applications, particularly in the field of information storage. Small magnetic particles are promising candidates for a further increase of the density of magnetic storage devices towards $100 \mathrm{Gbit} / \mathrm{inch}^{2}$ up to a few Tbit/inch ${ }^{2}$ [122]. Apart from data storage, there are potential other applications of 


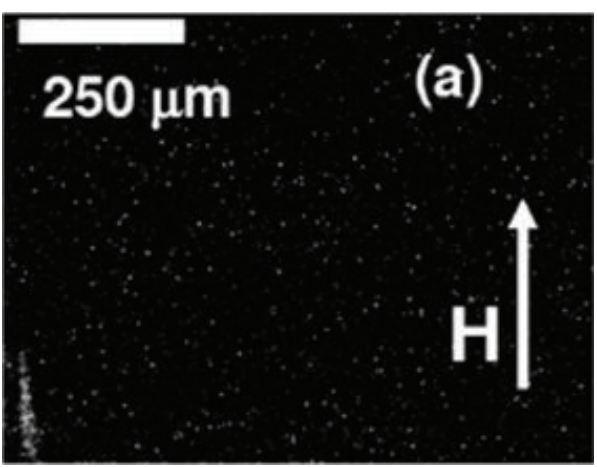

(a)

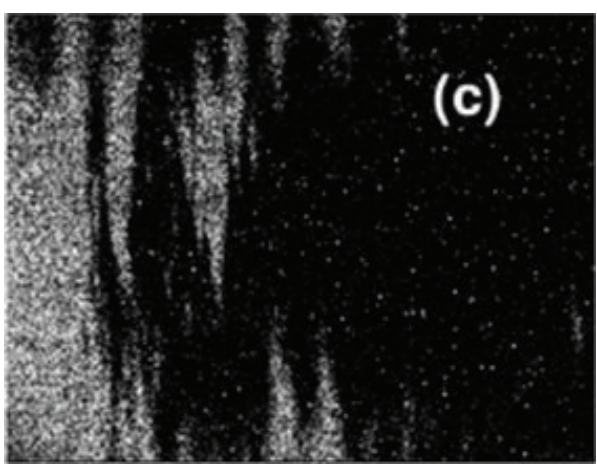

(c)

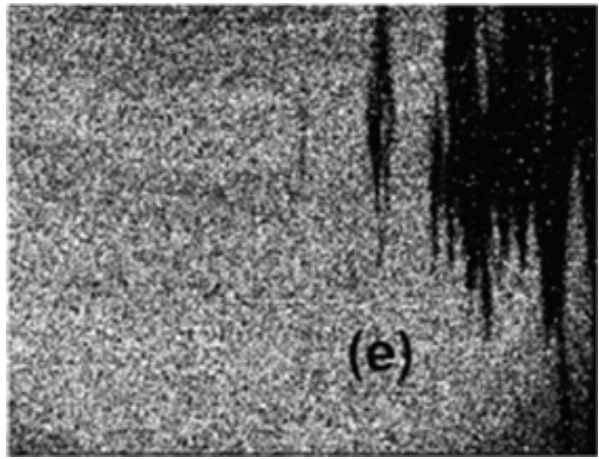

(e)

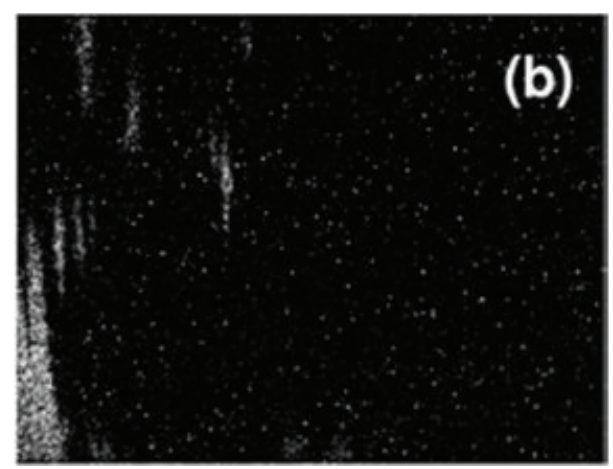

(b)

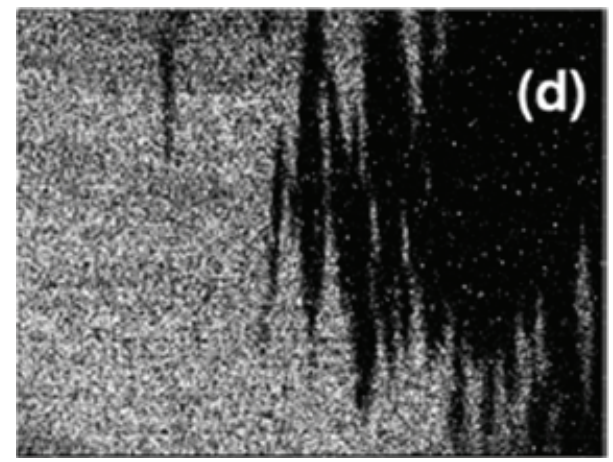

(d)

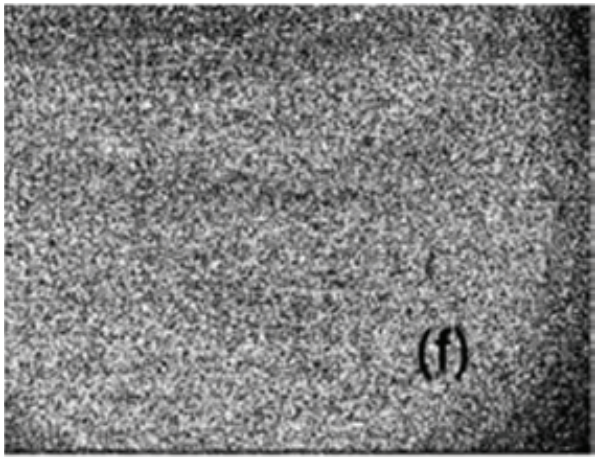

(f)

FIGURE 12: Longitudinal MOKE microscopy images of superferromagnetic $\left[\mathrm{Co}_{80} \mathrm{Fe}_{20}(1.3 \mathrm{~nm}) / \mathrm{Al}_{2} \mathrm{O}_{3}(3 \mathrm{~nm})\right]_{10}$ taken at room temperature under supercoercive fields, $\mu_{0} H=0.6 \mathrm{mT}$, at $t=2 \mathrm{~s}(\mathrm{a}), 3 \mathrm{~s}(\mathrm{~b}), 4 \mathrm{~s}(\mathrm{c}), 5 \mathrm{~s}(\mathrm{~d}), 6 \mathrm{~s}(\mathrm{e})$, and $9 \mathrm{~s}(\mathrm{f})$. Reproduced with permission from Bedanta et al. [20] () 2010, IOP Publishing.

magnetic nanoparticles, for example, in ferrofluids, highfrequency electronics, high performance permanent magnets, and magnetic refrigerants. Magnetic particles are also potential candidates to be used in biology and medical applications such as drug-targeting, cancer therapy, lymph node imaging, or hyperthermia $[10,11,123]$.

In recent years, researchers have tried to fabricate MNP based multifunctional nanostructures. There are basically two types of approaches: (i) molecular functionalization, which involves attaching the magnetic nanoparticles to antibodies, proteins and dyes, and so forth, and (ii) integration of MNPs with other functional nanoparticles, such as quantum dots or metallic nanoparticles [23]. For example, semiconducting chalcogenides have been grown by using magnetic nanoparticles as seeds. In this case, the final product is core-shell or heterodimer nanostructures with both magnetic and fluorescent properties. This leads to the demonstration of intracellular manipulation of nanoparticles and a promising candidate for dual-functional molecular imaging (i.e., combined MRI and fluorescence imaging). MNPs can be used as MRI contrast enhancement agents, since the signal of magnetic moments of protons around magnetic nanoparticles can be captured by resonant absorption [24]. These multifunctional MNPs could be used in biological 
applications such as protein purification, bacteria detection, and toxin decorporation [23]. Figure 13 illustrates these two approaches for making multifunctional MNPs and their various biological applications.

In the last three decades, magnetic data storage has seen a linear rise in terms of storage capacity. The physics of magnetic nanostructures is at the heart of magnetic hard disk drive technology. In the future, it is very probable that areal densities will increase well beyond 1 Terabit/inch ${ }^{2}$ by employing new technologies like bit patterned media (BPM) or heat assisted magnetic recording $[122,124]$.

Patterned magnetic nanostructures, such as twodimensional dot-arrays have attracted the interest of researchers due to their potential applications such as magnetic information storage [125] or nonvolatile magnetic random access memory (MRAM) [126]. The demand for ultrahigh density magnetic storage devices drives the bit size into the nanometer scale. As the volume $V=\pi D^{2} t / 4$ (where $D$ and $t$ are the diameter and thickness, resp.) of the grains is reduced in the scaling process, the magnetization of the grains may become unstable due to thermal fluctuations, and data loss may occur [122]. As the physical size of the nanostructures in the patterned array decreases, loss of data due to the thermal instability (also known as "superparamagnetic (SPM) effect") would become a very crucial issue [127]. Therefore, future data storage technology has to overcome the SPM effect. In this regard, the $L 1_{0}-\mathrm{FePt}$ alloy is one of the most promising materials for future ultrahigh density magnetic storage devices because it possesses a huge uniaxial magnetocrystalline anisotropy $\left(K_{u}=7 \times 10^{7} \mathrm{erg} / \mathrm{cc}\right)$ [128], which leads to a high thermal stability of magnetization.

Also the present longitudinal data storage media may be considered as a collection of independent particles because of their weak intergranular exchange coupling. However, as we have discussed in the superferromagnetic section (Section 7), strong intergranular interactions can drive the system to form long-range ordered SFM domains, which are clearly unsuitable for applications in data storage. Also the SFM alignment counteracts large tunneling magnetoresistance (TMR) values, so magnetic random access memory applications are not promising for SFM systems. However, SFM materials are soft magnetics, which make them nearly ideal materials for high permeability, low-loss materials for microelectronics, power management, and sensing devices designed for high frequencies.

\section{Simulations and Modeling of Magnetic Nanostructures}

It is well known that numerical simulations constitute the third pillar of condensed matter physics besides experimental exploration and analytical theoretical description. With simulations, one models either the static spin structure in equilibrium or the spin dynamics as function of time or frequency. In the context of MNPs, usually two types of numerical simulations are employed, namely, micromagnetic [129] and Monte-Carlo simulations [130].
In micromagnetic simulations, the system under study is considered in the "continuum approximation" [17]. Instead of assuming localized moments, the magnetization is rather described by a continuous vector field $\mathbf{M}(\mathbf{r})$. For the practical simulation, the system has to be subdivided into cells. The cell size should be smaller than the exchange length of the material. Typical cell sizes are in the order of a few nanometers. Before the start, a certain user-defined initial magnetization configuration is chosen. Then, during the run of the simulation, the magnetization of each cell is updated based on a physical model. For micromagnetic simulations, this is (in most cases) the Landau-Lifshitz-Gilbert equation, which is an equation of motion of the magnetization vector in an effective field $[130,131]$ :

$$
\frac{d \mathbf{M}_{i}}{d t}=-\gamma^{\prime} \mathbf{M}_{i} \times \mathbf{H}_{\mathrm{eff}}-\frac{\alpha \gamma^{\prime}}{\mathbf{M}_{s}} \mathbf{M}_{i} \times\left(\mathbf{M}_{i} \times \mathbf{H}_{\mathrm{eff}}\right),
$$

where $\mathbf{H}_{\mathrm{eff}}=H+H_{\mathrm{demag}}+H_{K}+H_{\mathrm{ex}}+h(t)$ is the effective magnetic field with contributions from Zeeman, demagnetizing, anisotropy, exchange, and time-dependent magnetic fields and $\mathbf{M}_{i}$ is the magnetization of cell $i, \gamma^{\prime}=$ $\gamma /\left(1+\alpha^{2}\right)$, with $\gamma$ being the gyromagnetic ratio, $\mathbf{M}_{s}=$ saturation of magnetization of the material under study and $\alpha$ the damping constant of the specific system. All energy contributions like Zeeman energy, anisotropy energy, and interactions act in the form of an effective field $\mathbf{H}_{\text {eff }}$ onto the magnetization vector [129]. Since micromagnetic simulations are explicitly referring to $T=0$, they are usually employed to larger nanomagnets, where thermal fluctuations are less relevant to calculations of the ground state at low enough temperatures.

Contrastingly, if the temperature behavior of MNPs has to be calculated, Monte-Carlo simulations are used [130, 132, 133]. In this type of simulations, the system is considered in the model of localized moments. Hence, each moment is updated in the course of the simulation. The updating is based on Monte-Carlo techniques. The most popular approach is hereby the Metropolis algorithm [130], which calculates the statistical probability of a spin-flip or rotation based on the comparison of a random number with the Boltzmann factor. For the calculation of the Boltzmann factor, the Hamiltonian of the system needs to be known.

\section{Magnetization Dynamics and LLG Modeling of Magnetic Nanoparticles}

The quasistatic and ultrafast magnetic properties of magnetic nanostructures are different from their bulk counterparts. Magnetization dynamics of these systems strongly depends upon their magnetic ground states, which depend not only on their intrinsic material parameters such as exchange stiffness constant, saturation magnetization, and magnetocrystalline anisotropy but also on their physical structures as well as the external parameters such as the strength and orientation of the bias magnetic field. To study the quasistatic and ultrafast dynamic properties of nanomagnets, different kinds of sensitive characterization techniques have been developed in last few decades. Electron and force based 


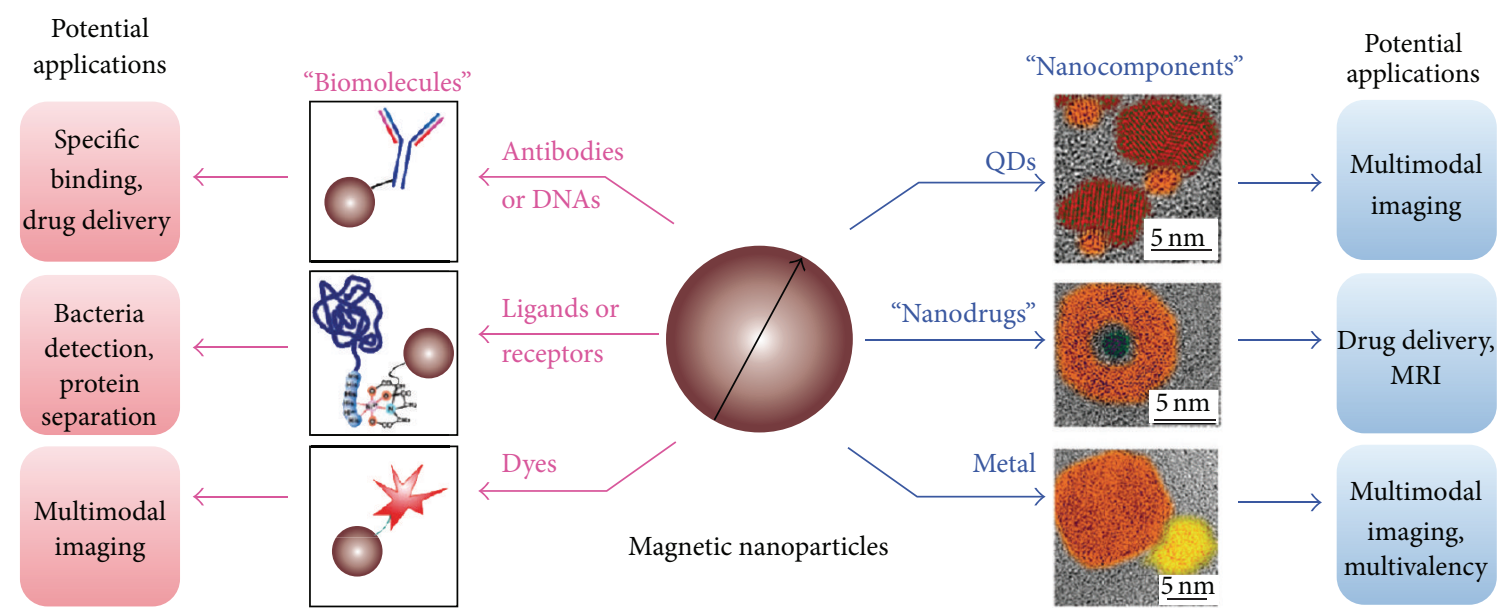

FIGURE 13: Various potential applications of multifunctional magnetic nanoparticles in biology. Reproduced with permission from Gao et al. [24] (c) 2009, American Chemical Society.

magnetic microscopy such as magnetic force microscopy (MFM), Lorentz force microscopy (LFM), photoemission electron microscopy (PEEM), spin polarized low energy electron microscopy (SPLEEM), scanning electron microscopy with polarization analysis (SEMPA), spin polarized scanning tunneling microscopy (SP-STM), electron holography, and ballistic electron magnetic microscopy all provide excellent spatial resolution, but offer very poor or moderate temporal resolution.

Consequently, different kinds of techniques have emerged to investigate the fast magnetization dynamics of magnetic thin films and confined magnetic structures. Out of those techniques, ferromagnetic resonance (FMR) and Brillouin light scattering (BLS) provide information on the dynamics in the frequency and wave-vector domain, respectively. Recent developments of spatially resolved FMR [134] and BLS [135] techniques have emerged as powerful tools to study the profiles of the dynamic modes in confined magnetic structures. The magnetoresistive method [136], X-ray microscopy [137], and pulse inductive magnetometry [138] also have the potential to emerge as powerful techniques. On the other hand the time-resolved magneto-optical Kerr effect has emerged as one of the most powerful techniques to study the femto- and picosecond magnetization dynamics of magnetic nanostructures due to simultaneous spatiotemporal resolution. Here, we will review the time-resolved study of femto- and picosecond magnetization dynamics of magnetic nanostructures.

11.1. Background Theory. Magnetization dynamics can occur over a wide range of timescales. Laser induced ultrafast demagnetization occurs within a few hundreds of femtoseconds. The fast remagnetization time following the ultrafast demagnetization covers the time scale of 1-10 picoseconds. The precession of magnetization occurs within few picoseconds to few hundreds of picoseconds. The damping of magnetization precession occurs on sub-nanosecond to tens of nanoseconds time scales. The slowest process is the domain wall dynamics, which occurs between a few nanoseconds and microseconds.

The time evolution of magnetization under the application of a time-dependent magnetic field $h(t)$ may be modeled by the Landau-Lifshitz-Gilbert equation of motion $[130,131]$ given by (5).

Under the macrospin model and assuming a uniform ellipsoidal particle with demagnetizing tensor axes $N_{x}, N_{y}$, and $N_{z}\left(N_{x}+N_{y}+N_{z}=4 \pi\right)$, Kittel [13] derived the frequency for the uniform precessional mode, also known as ferromagnetic resonance mode as $\omega_{0}=\gamma\left(\left[H_{z}+\left(N_{y}-N_{z}\right) M_{z}\right] \times\left[H_{z}+\left(N_{x}-N_{z}\right) M_{z}\right]\right)^{1 / 2}$. If the system has two- and fourfold magnetic anisotropies given by the energies $F_{2}=-K_{2} \sin \theta \cos ^{2} \phi$ and $F_{4}=\left(K_{4} / 4\right)$ $\sin ^{4} \theta(3+\cos 4 \phi)$, the resonant frequencies will take forms as

$$
\begin{aligned}
& \omega_{0}=\gamma( {\left[H_{z}+\left(N_{y}-N_{z}+\frac{2 K_{2}}{M_{s}} \cos 2 \phi\right) M_{z}\right] } \\
&\left.\times\left[H_{z}+\left(N_{x}-N_{z}+\frac{2 K_{2}}{M_{s}} \cos ^{2} \phi\right) M_{z}\right]\right)^{1 / 2}, \\
& \omega_{0}=\gamma\left(\left[H_{z}+\left(N_{y}-N_{z}-\frac{4 K_{4}}{M_{s}} \cos 4 \phi\right) M_{z}\right]\right. \\
&\left.\times\left[H_{z}+\left(N_{x}-N_{z}-\frac{K_{4}}{M_{s}}(3+\cos 4 \phi)\right) M_{z}\right]\right)^{1 / 2} .
\end{aligned}
$$

These equations are valid for the case of vanishing wavevector, $k=0$. For finite wave-vector spin waves a dispersion of the spin wave frequencies with the wave-vector can be found. In the exchange dominated regime (very large $k$ ), $\omega(k)$ is proportional to $(1-\cos k a)$ and in the long wavelength limit $(k a \ll 1)$, this becomes $\omega(k)=\gamma\left(H+D k^{2}\right)$, where 
$D=2 A / M_{s}$ and $A$ is the exchange stiffness constant. The perpendicular standing spin wave (PSSW) mode observed in a thin film is an example of exchange spin wave. The spin wave propagates along the thickness of the film and reflects back to form a standing spin wave. The wave-vector is quantized for pinned or unpinned boundary condition. The wave-vector can have values $n(\pi / d)$, where $d$ is the film thickness and $n$ is a positive integer.

If an in-plane magnetic field is applied to an infinite ferromagnetic thin film, long wavelength spin waves $(\lambda \sim 1 \mu \mathrm{m})$ dominated by dipolar interaction are observed. The dispersion relations of dipolar modes can be calculated numerically by solving the Landau-Lifshitz equation after considering Maxwell's equations in the magnetostatic limit. The dispersion relation of dipole-exchange spin wave in an infinite ferromagnetic material can be written by the HerringKittel formula [139]:

$$
\omega=\gamma\left[\left(H+\frac{2 A}{M_{s}} k^{2}\right)\left(H+\frac{2 A}{M_{s}} k^{2}+4 \pi M_{s} \sin ^{2} \theta_{k}\right)\right]^{1 / 2},
$$

where $\theta_{k}$ is the angle between $k$ and $M_{s}$. Damon and Eshbach [140] first calculated the dispersion of dipolar modes for a thin magnetic film. They found two kinds of solutions: the surface or Damon-Eshbach (DE) and the volume mode. In general, when the surface mode propagates perpendicularly to the magnetization, it is called magnetostatic surface wave (MSSW) mode. Considering negligible anisotropy, the dispersion relation of MSSW mode is given by

$$
\omega_{\mathrm{DE}}=\gamma\left[H\left(H+4 \pi M_{s}\right)+\left(2 \pi M_{s}\right)^{2}\left(1-e^{-2 k d}\right)\right]^{1 / 2} .
$$

There is a manifold of volume or bulk modes. When the directions of $\mathbf{M}$ and $\mathbf{k}$ are identical and both lie in the plane of the film, the spin wave is called the backward volume magnetostatic mode (BWVMS). Considering negligible anisotropy the dispersion relation of lowest order BWVMS spin waves is given by

$$
\left(\frac{\omega_{B}}{\gamma}\right)^{2}=H\left[H+4 \pi M_{s}\left(\frac{1-e^{-2 k d}}{k d}\right)\right] .
$$

The negative slope of the dispersion implies that the phase velocity and group velocity are in opposite directions. When the magnetization is along the normal to the film and propagation direction is in the plane of the film, a forward volume magnetostatic (FWVMS) mode is observed. The dispersion relation can be expressed after neglecting anisotropy as

$$
\left(\frac{\omega_{F}}{\gamma}\right)^{2}=\left(H-4 \pi M_{s}\right)\left[H-4 \pi M_{s}\left(\frac{1-e^{-k d}}{k d}\right)\right] .
$$

In a confined magnetic structure spin waves can also be quantized in the plane of the film. If $w$ is the width of nanostructure, then the values of quantized wave-vector spin waves may be written as $k_{n}=2 \pi / \lambda_{n}=n \pi / w$. The nonuniform demagnetization field and the edge effect must be considered to calculate the confined spin wave modes. A convenient alternative to calculate the quantized spin waves is solving the Landau-Lifshitz equation (5) within the framework of micromagnetism.
11.2. Time-Resolved Magneto-Optical Kerr Effect Measurements. Time-resolved magneto-optical Kerr effect measurements are based upon an optical pump-probe experiment. In its most general form, a laser pulse (typical pulse width of $100 \mathrm{fs}$ ) is divided into a strong pump beam and a weak probe beam by a beam splitter. The pump is used to excite the magnetization dynamics, while the probe is time-delayed with respect to the pump beam by an optical delay generator and is used to probe the dynamics in a noninvasive manner by measuring the magneto-optical Kerr (or Faraday) effect. Upon reflection (transmission) from the sample the plane polarized probe beam becomes elliptically polarized, where the rotation angle or ellipticity gives a measure of the magnetization state of the sample. In some cases, second harmonic MOKE [141] is also used, which is highly sensitive at the surface of thin film samples. The Kerr (Faraday) rotation/ellipticity is measured by either using a photoelastic modulator and an analyzer or a balanced photodiode detector [142], both of which provide very sensitive detection of rotation and ellipticity, respectively, down to microdegrees. The time-delay between the pump and probe beams is scanned and the Kerr (Faraday) rotation/ellipticity is measured at each scan point to build the time evolution of magnetization after excitation by the pump pulse. The excitation can be electronic or purely optical. In 1991, Freeman et al. [143] reported the first time-resolved magneto-optical Kerr effect measurement of magnetization evolution and relaxation dynamics in pure and $\mathrm{Tb}$ doped EuS thin film. They used an optically triggered photoconductive switch [144] to generate a current pulse, which flows through a transmission line structure to produce a pulsed magnetic field. The pulsed magnetic field excites the dynamics and the corresponding relaxation is studied. Later in 1992 [145], they showed clear precessional dynamics in an yttrium iron garnet (YIG) film using the same technique. Using this technique, Elezzabi et al. [146] reported the direct measurement of conduction electron spin-lattice relaxation time T1 in gold film as $45 \pm 5$ ps suggesting that Matthiessen's rule is not obeyed for conduction electron spin scattering. In the same year, Beaurepaire et al. [147] demonstrated subpicosecond demagnetization of metallic Ni film by direct excitation with a femtosecond laser pulse. The measurement also allowed to deduce electron and spin temperatures and gives a value to the electron-spin coupling constant. In 1997, the first time-resolved stroboscopic imaging of nonuniform precessional dynamics in a microscale permalloy dot was reported [148]. These works triggered a flurry of experimental and theoretical works in the investigation of optical and field pump induced spin and magnetization dynamics in magnetic thin films and patterned structures. Even though doubts were raised on whether the optically induced ultrafast magnetooptics in Ni is pure magnetism or optics [149], van Kampen et al. demonstrated an all-optical method to excite and detect coherent spin waves in magnetic materials in 2002 [150]. The approach is based upon the temperature dependence of the anisotropy, which allows one to use the heat from an absorbed laser pulse to generate a fast anisotropy field pulse, triggering a precession of magnetization. 
11.3. Magnetization Dynamics of Nanomagnets. The initial works on the magnetization dynamics of nanomagnets have started to appear in early 2000 by FMR [151] and BLS techniques [152]. The first time-resolved measurement of precessional dynamics in nanomagnet arrays induced by magnetic field pulse was reported by Kruglyak et al. in 2005 [153], where they showed a size dependent variation of precession frequency and a cross-over to nonuniform precession as the size of the permalloy nanodots is reduced to below $220 \mathrm{~nm}$. These experiments were performed on arrays of nanomagnets, where the magnetostatic-interaction effects from the neighboring elements cannot be ignored. In 2006, Barman et al. [154] used a novel technique known as the time-resolved cavity enhanced MOKE to study the intrinsic femto- and picosecond dynamics of single cylindrical shaped $\mathrm{Ni}$ nanomagnets with diameter varying from $5 \mu \mathrm{m}$ down to $125 \mathrm{~nm}$. The idea was based upon enhancing the magneto-optical Kerr rotation by coating the nanomagnets with a dielectric enhancement layer. By properly choosing the thickness and material index of the dielectric layer, the Kerr signal reflected off the magnetic surface was enhanced by five times through constructive multiple reflections. A very interesting size dependence of precession frequency was observed due to the variation of magnetic ground states from in-plane multidomain to vortex and finally to out-of-plane magnetized quasisingle domain state (Figure 14). The damping also showed a strong size dependence with a transition to a small damping value at the nanoscale as opposed to a large value at micron and submicron scales [155]. Later, a dynamic configurational anisotropy was shown in arrays of $220 \mathrm{~nm}$ permalloy nanomagnets [156] originating from the variation of both the static and dynamic magnetization configuration and the associated dynamic effective magnetic field. In 2007, Laraoui et al. [157] presented ultrafast thermal switching, relaxation, and precession of individual $\mathrm{CoPt}_{3}$ nanodisks and permalloy microdisks with diameter down to $200 \mathrm{~nm}$. A coherent thermal switching using $8 \mathrm{~mJ} / \mathrm{cm}^{2}$ pump pulses and a bi-exponential relaxation in $\mathrm{CoPt}_{3}$ was observed. A fast remagnetization $\left(\tau_{\text {spin-lat }}=5.2 \mathrm{ps}\right)$ is associated with the equilibrium between the spin and lattice, while the slow remagnetization $\left(\tau_{\text {diff }}=530 \mathrm{ps}\right)$ corresponds to the thermal diffusion to the surrounding of the disk deposited on a sapphire substrate. In 2008, Liu et al. [158] showed that the shape of the nanodisks significantly affects the vortex-toquasisingle domain state transitions and the time-resolved magnetization dynamics showed different modal frequencies due to the transition between different domain states. They observed that this transition can also be spontaneously triggered, when the bias field is kept fixed at a critical field region and the disk is driven into dynamics. In 2008, Keatley et al. [159] showed that at large amplitude precession the edge mode can be suppressed and dynamics is dominated by the center mode, which is a useful result from the viewpoint of nanoscale spin transfer torque oscillators and bistable switching devices, where large amplitude dynamics happens.

11.4. Collective Magnonic Modes in Arrays of Nanomagnets. An array of dipolar coupled nanomagnets may show long wavelength collective dynamics, where the dynamics of the constituent nanomagnets maintain constant amplitude and phase relationships similar to the acoustic and optical modes of phonons. These long wavelength collective dynamics in the form of Bloch waves, defined in the Brillouin zone (BZ) of an artificial lattice, can be manipulated by tailoring the lattice to form magnonic crystals, the magnetic analogue of photonic, and phononic crystals.

In 2010, Kruglyak et al. [160] reported the measurement and imaging of collective magnonic modes in arrays of $80 \times$ $40 \mathrm{~nm}^{2} \quad \mathrm{Co}_{50} \mathrm{Fe}_{50}(0.7 \mathrm{~nm}) / \mathrm{Ni}_{92} \mathrm{Fe}_{8}(4: 5 \mathrm{~nm})$ elements with $20 \mathrm{~nm}$ interdot separation. The pulsed field excited dynamics showed a broad single peak at higher bias field and splitting into three narrower peaks at smaller bias field values due to the appearance of the collective nonuniform precessional modes such as quasiuniform and backward volume-like and Damon-Eshbach-like modes. In 2011, Rana et al. [161] showed an all-optical excitation and detection of collective modes in arrays of $200 \mathrm{~nm}$ square dots with varying interdot separation (S) from $400 \mathrm{~nm}$ down to $50 \mathrm{~nm}$. The dynamics showed a systematic transition from a strongly collective regime $(S \leq$ $75 \mathrm{~nm})$ to a weakly collective regime $(100 \mathrm{~nm} \leq S \leq 300 \mathrm{~nm})$ to a completely isolated regime $(S \geq 400 \mathrm{~nm})$. An anisotropy of the strongly collective mode $(S=50 \mathrm{~nm})$ was observed when the orientation of the bias field is rotated with respect to the lattice symmetry [162]. In addition to a fourfold symmetry of the mode frequency, a transition from a strongly collective to an isolated regime was also observed as the bias field is rotated from 0 to $45^{\circ}$ with respect to the symmetry axis. In 2011, Rana et al. [163] showed the detection of $50 \mathrm{~nm}$ permalloy dots in the single nanodot regime, where the dots are magnetostatically isolated (Figure 15). The dynamics of the isolated $50 \mathrm{~nm}$ permalloy dot is dominated by the edge mode, while the center mode becomes almost nonexistent. The damping of this mode is close to the thin film value. When these dots are arranged in arrays, the frequencies of the modes increase with the decrease in interdot separation primarily due to the quadrupolar interaction followed by a mode splitting, where a collective-like backward volumelike mode appears in addition to the coherent mode of the array. The damping also shows an increase with the decrease in interdot separation due to the dynamic dephasing of the dots predicted earlier in 2009 [164]. In 2013, Saha et al. [165] showed how the collective magnonic modes of a twodimensional nanomagnetic lattice can be tuned by the lattice symmetry. The interdot magnetostatic interaction is tuned by varying the lattice symmetry, which results in new collective magnonic modes as the symmetry reduces from square to octagonal through rectangular, hexagonal, and honeycomb symmetry. Further works on nanodots with different shapes $[166,167]$ have shown various anisotropic spin waves, which can form building blocks for two-dimensional magnonic crystals.

The collective magnetization dynamics of arrays of magnetic nanodots is an important problem with future prospects of applications in on-chip microwave communication and spin-logic devices. Magnonic band formation, tunability of bandgaps, control on propagation velocity, anisotropy 

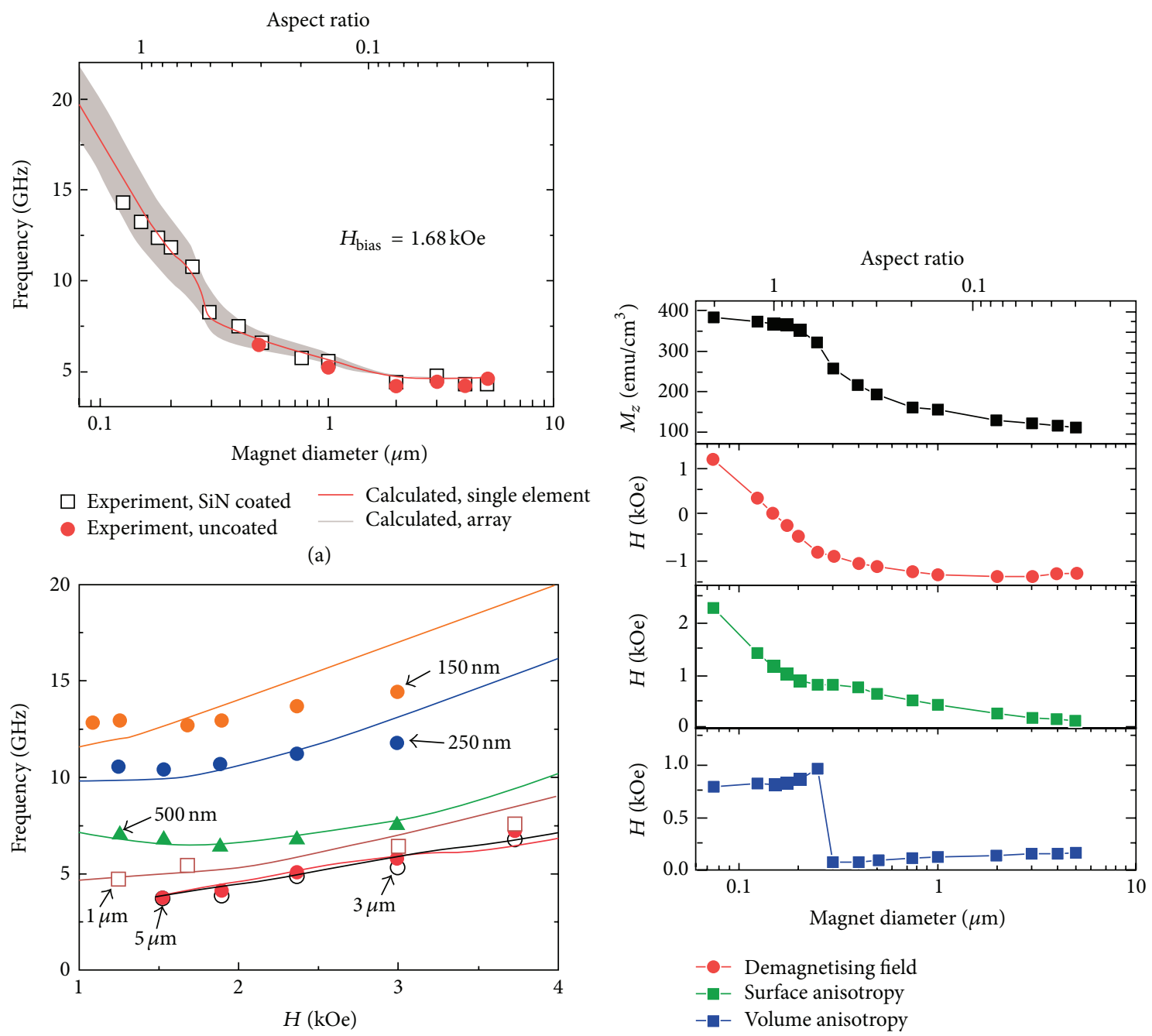

(b)

(c)

Figure 14: Theoretical modeling of the diameter and bias-field dependence of experimentally obtained precession frequencies. (a) Experimentally obtained frequencies of the uniform precession modes from uncoated and $70 \mathrm{~nm}$ SiN-coated magnets as a function of magnet diameter (aspect ratio) at $H_{\text {bias }} 1.68 \mathrm{kOe}$. Solid line: calculated precession frequencies of single magnets from (1). The gray shaded region shows the possible range of precession frequencies in arrays of magnets separated by $56 \mathrm{~nm}$. (b) Experimental (points) and calculated (curves) precession frequencies as a function of bias magnetic field $\left(H_{\text {bias }}\right)$. (c) $M_{z}$ and contributions of different contributions to the effective magnetic field as a function of magnet diameter. Reproduced with permission from Barman et al. [154] ๔ 2006, American Chemical Society.

in the spin wave propagation, and variation in Gilbert damping and extrinsic contributions to the damping are some of the important issues to be dealt with. Hence, more detailed understanding of the spin wave dynamics in magnetic nanodot arrays and possible control of above properties would open up exciting new prospects in these fields. On the other hand, further development of powerful techniques such as time-resolved near field MOKE and Xray microscopy for studying magnetization dynamics with very high spatiotemporal resolution will enable the study of the intrinsic dynamics of smaller single nanomagnets as well as their dynamics under the magnetostatic interaction of the neighboring elements when placed in arrays of various lattice constants and lattice symmetries.

\section{Summary and Outlook}

We have discussed various aspects concerning the research on MNPs and nanostructures. The research on synthesis of magnetic nanoparticles has made a significant progress in particular with chemical routes. By coating the MNPs with organic ligands, the agglomeration and oxidation issue can be taken care of easily. Lithography techniques have seen significant developments for making patterning of nanostructures and in particular of magnetic materials for future data storage. We have discussed that nanoparticle assemblies may show different magnetic states such as SPM, SSG, or SFM depending on the strength and symmetry of interparticle interactions. While the structural characterization of nanoparticle assemblies is usually performed 


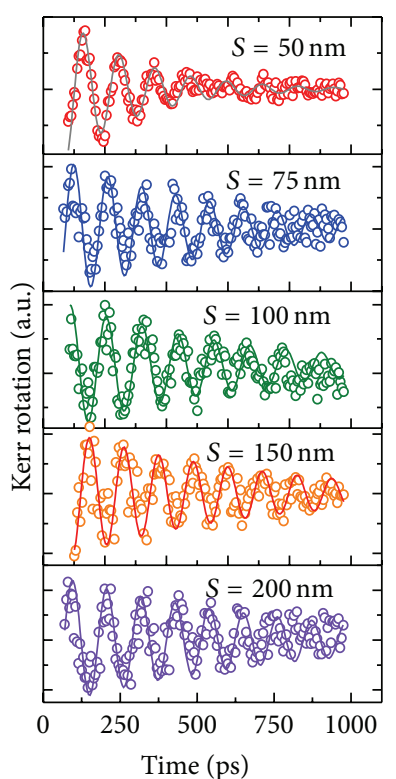

(a)

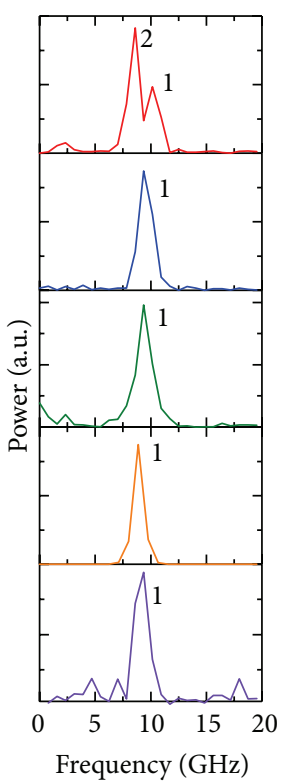

(b)

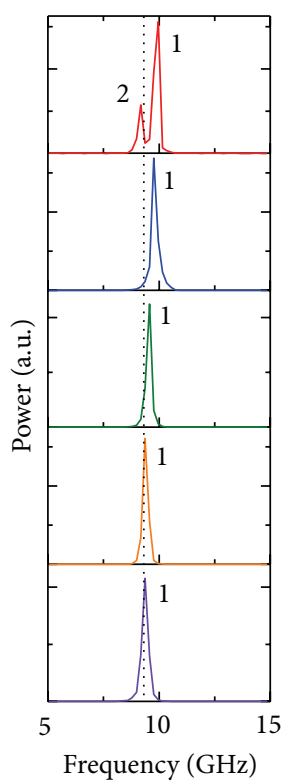

(c)

FIGURE 15: (a) Experimental time-resolved Kerr rotations and (b) the corresponding FFT spectra are shown for arrays of permalloy dots with width $=50 \mathrm{~nm}$, thickness $=20 \mathrm{~nm}$, and with varying interdot separation $S$ at $H=2.5 \mathrm{kOe}$. (c) The FFT spectra of the simulated time-resolved magnetization are shown. The peak numbers are assigned to the FFT spectra. The dotted line in (c) shows the simulated precession frequency of a single permalloy dot with width $=50 \mathrm{~nm}$ and thickness $=20 \mathrm{~nm}$. Reproduced with permission from Rana et al. [163] (c) 2011, American Chemical Society.

with transmission electron, scanning electron or atomic force microscopy, X-ray diffraction, and so forth, the magnetic states are characterized by different techniques such as SQUID magnetometry, ac susceptometry, Mössbauer or muon spectroscopy, neutron scattering, and magnetic force microscopy.

Still the field of magnetic nanoparticles has several open challenges, which should be addressed in the next couple of years.

(i) Study of the Curie temperature of nanomagnets.

(ii) Synthesis of monodisperse MNPs with wellcontrolled shape, size, and crystallinity.

(iii) Study of supracrystals in order to understand the origin of $3 \mathrm{D}$ ordering.

(iv) Study of SSG correlations.

(v) Study of SFM systems with different magnetic materials, particle sizes, and so forth.

(vi) Understanding the origin of superferromagnetism: can SFM be observed in a purely dipolarly coupled system?

(vii) Getting insight into the SFM domain wall picture: are they real and do they have finite width?

(viii) Understanding the dynamics of nanomagnets by spectroscopy and micromagnetic modeling going for applications in data storage and biology.

\section{Conflict of Interests}

The authors declare no financial conflict of interests.

\section{References}

[1] S. Tehrani, E. Chen, M. Durlam et al., "High density submicron magnetoresistive random access memory (invited)," Journal of Applied Physics, vol. 85, no. 8, pp. 5822-5827, 1999.

[2] T. Thomson, G. Hu, and B. D. Terris, "Intrinsic distribution of magnetic anisotropy in thin films probed by patterned nanostructures," Physical Review Letters, vol. 96, no. 25, Article ID 257204, 4 pages, 2006.

[3] J. R. Childress and R. E. Fontana Jr., "Magnetic recording read head sensor technology," Comptes Rendus Physique, vol. 6, no. 9, pp. 997-1012, 2005.

[4] S. H. Chung, A. Hoffmann, S. D. Bader et al., "Biological sensors based on Brownian relaxation of magnetic nanoparticles," Applied Physics Letters, vol. 85, no. 14, pp. 2971-2973, 2004.

[5] M. Arruebo, R. Fernández-Pacheco, M. R. Ibarra, and J. Santamaría, "Magnetic nanoparticles for drug delivery," Nano Today, vol. 2, pp. 22-32, 2007.

[6] D. A. Allwood, G. Xiong, C. C. Faulkner, D. Atkinson, D. Petit, and R. P. Cowburn, "Magnetic domain-wall logic," Science, vol. 309, no. 5741, pp. 1688-1692, 2005.

[7] A. Imre, G. Csaba, L. Ji, A. Orlov, G. H. Bernstein, and W. Porod, "Majority logic gate for magnetic quantum-dot cellular automata," Science, vol. 311, no. 5758, pp. 205-208, 2006.

[8] S. Kaka, M. R. Pufall, W. H. Rippard, T. J. Silva, S. E. Russek, and J. A. Katine, "Mutual phase-locking of microwave spin torque nano-oscillators," Nature, vol. 437, no. 7057, pp. 389-392, 2005. 
[9] B. Lenk, H. Ulrichs, F. Garbs, and M. Münzenberg, "The building blocks of magnonics," Physics Reports, vol. 507, no. 4-5, pp. 107-136, 2011.

[10] C. C. Berry and A. S. G. Curtis, "Functionalisation of magnetic nanoparticles for applications in biomedicine," Journal of Physics D, vol. 36, no. 13, pp. R198-R206, 2003.

[11] P. Tartaj, M. del Puerto Morales, S. Veintemillas-Verdaguer, T. González-Carreño, and C. J. Serna, "The preparation of magnetic nanoparticles for applications in biomedicine," Journal of Physics D, vol. 36, no. 13, pp. R182-R197, 2003.

[12] J. Frenkel and J. Dorfman, "Spontaneous and induced magnetisation in ferromagnetic bodies," Nature, vol. 126, no. 3173, pp. 274-275, 1930.

[13] C. Kittel, "Theory of the structure of ferromagnetic domains in films and small particles," Physical Review, vol. 70, no. 11-12, pp. 965-971, 1946.

[14] E. C. Stoner and E. P. Wohlfarth, "A mechanism of magnetic hysteresis in heterogeneous alloys," IEEE Transactions on Magnetics, vol. 27, no. 4, pp. 3475-3518, 1991.

[15] R. C. O'Handley, Modern Magnetic Materials: Principles and Applications, Wiley-VCH, Weinheim, Germany, 2000.

[16] D. Givord, Q. Lu, and M. F. Rossignol, Science and Technology of Nanostructured Materials, G. C. Hadjipanayis and G. A. Prinz, Eds., Plenum Press, New York, NY, USA, 1991.

[17] S. Blundell, Magnetism in Condensed Matter, Oxford University Press, New York, NY, USA, 2001.

[18] E. C. Stoner and E. P. Wohlfarth, "A mechanism of magnetic hysteresis in heterogeneous alloys," Philosophical Transactions of the Royal Society A, vol. 240, pp. 599-642, 1948.

[19] T. Hyeon, "Chemical synthesis of magnetic nanoparticles," Chemical Communications, vol. 9, no. 8, pp. 927-934, 2003.

[20] C. B. Murray, D. J. Norris, and M. G. Bawendi, "Synthesis and characterization of nearly monodisperse $\mathrm{CdE}(\mathrm{E}=\mathrm{S}$, Se, $\mathrm{Te}$ ) semiconductor nanocrystallites," Journal of the American Chemical Society, vol. 115, no. 19, pp. 8706-8715, 1993.

[21] A.-H. Lu, E. L. Salabas, and F. Schüth, "Magnetic nanoparticles: synthesis, protection, functionalization, and application," Angewandte Chemie (International Edition), vol. 46, no. 8, pp. 12221244, 2007.

[22] S. Singamaneni, V. N. Bliznyuk, C. Binek, and E. Y. Tsymbal, "Magnetic nanoparticles: recent advances in synthesis, selfassembly and applications," Journal of Materials Chemistry, vol. 21, no. 42, pp. 16819-16845, 2011.

[23] L. E. Euliss, S. G. Grancharov, S. O’Brien et al., "Cooperative assembly of magnetic nanoparticles and block copolypeptides in aqueous media," Nano Letters, vol. 3, no. 11, pp. 1489-1493, 2003.

[24] J. Gao, H. Gu, and B. Xu, "Multifunctional magnetic nanoparticles: design, synthesis, and biomedical applications," Accounts of Chemical Research, vol. 42, no. 8, pp. 1097-1107, 2009.

[25] U. Jeong, X. Teng, Y. Wang, H. Yang, and Y. Xia, "Superparamagnetic colloids: controlled synthesis and niche applications," Advanced Materials, vol. 19, no. 1, pp. 33-60, 2007.

[26] S. Laurent, D. Forge, M. Port et al., "Magnetic iron oxide nanoparticles: synthesis, stabilization, vectorization, physicochemical characterizations, and biological applications," Chemical Reviews, vol. 108, pp. 2064-2110, 2008.

[27] S. Sun, C. B. Murray, D. Weller, L. Folks, and A. Moser, "Monodisperse FePt nanoparticles and ferromagnetic FePt nanocrystal superlattices," Science, vol. 287, no. 5460, pp. 19891992, 2000.
[28] B. Jeyadevan, A. Hobo, K. Urakawa, C. N. Chinnasamy, K. Shinoda, and K. Tohji, "Towards direct synthesis of fct-FePt nanoparticles by chemical route," Journal of Applied Physics, vol. 93, no. 10, pp. 7574-7576, 2003.

[29] S. Momose, H. Kodama, T. Uzumaki, and A. Tanaka, "Magnetic properties of magnetically isolated $\mathrm{Ll}_{0}-\mathrm{FePt}$ nanoparticles," Applied Physics Letters, vol. 85, no. 10, pp. 1748-1750, 2004.

[30] S. Yamamoto, Y. Morimoto, T. Ono, and M. Takano, "Magnetically superior and easy to handle $L 1_{0}$-FePt nanocrystals," Applied Physics Letters, vol. 87, no. 3, Article ID 032503, 2005.

[31] H. Zeng, J. Li, J. P. Liu, Z. L. Wang, and S. Sun, "Exchangecoupled nanocomposite magnets by nanoparticle selfassembly," Nature, vol. 420, no. 6914, pp. 395-398, 2002.

[32] M. Albrecht, G. Hu, I. L. Guhr et al., "Magnetic multilayers on nanospheres," Nature Materials, vol. 4, no. 3, pp. 203-206, 2005.

[33] F. Q. Zhu, G. W. Chern, O. Tchernyshyov, X. C. Zhu, J. G. Zhu, and C. L. Chien, "Magnetic bistability and controllable reversal of asymmetric ferromagnetic nanorings," Physical Review Letters, vol. 96, no. 2, Article ID 027205, 4 pages, 2006.

[34] F. Ernult, S. Mitani, K. Takanashi et al., "Self-alignment of Fe nanoparticles on a tunnel barrier," Applied Physics Letters, vol. 87, no. 3, Article ID 033115, 2005.

[35] C. T. Black, C. B. Murray, R. L. Sandstrom, and S. Sun, "Spindependent tunneling in self-assembled cobalt-nanocrystal superlattices," Science, vol. 290, no. 5494, pp. 1131-1134, 2000.

[36] S. Mitani, S. Takahashi, K. Takanashi, K. Yakushiji, S. Maekawa, and H. Fujimori, "Enhanced magnetoresistance in insulating granular systems: evidence for higher-order tunneling," Physical Review Letters, vol. 81, no. 13, pp. 2799-2802, 1998.

[37] K. Yakushiji, F. Ernult, H. Imamura et al., "Enhanced spin accumulation and novel magnetotransport in nanoparticles," Nature Materials, vol. 4, no. 1, pp. 57-61, 2005.

[38] T. Shima, K. Takanashi, Y. K. Takahashi, and K. Hono, "Preparation and magnetic properties of highly coercive FePt films," Applied Physics Letters, vol. 81, no. 6, pp. 1050-1052, 2002.

[39] T. Shima, K. Takanashi, Y. K. Takahashi, and K. Hono, "Coercivity exceeding 100 kOe in epltaxially grown FePt sputtered films," Applied Physics Letters, vol. 85, no. 13, pp. 2571-2573, 2004.

[40] S. Okamoto, O. Kitakami, N. Kikuchi, T. Miyazaki, Y. Shimada, and Y. K. Takahashi, "Size dependences of magnetic properties and switching behavior in FePt $L 1_{0}$ nanoparticles," Physical Review B, vol. 67, no. 9, Article ID 094422, 2003.

[41] C. Pascal, J. L. Pascal, F. Favier, M. L. Elidrissi Moubtassim, and C. Payen, "Electrochemical synthesis for the control of $\gamma-\mathrm{Fe}_{2} \mathrm{O}_{3}$ nanoparticle size. Morphology, microstructure, and magnetic behavior," Chemistry of Materials, vol. 11, pp. 141-147, 1999.

[42] Z. Dai, F. Meiser, and H. Möhwald, "Nanoengineering of iron oxide and iron oxide/silica hollow spheres by sequential layering combined with a sol-gel process," Journal of Colloid and Interface Science, vol. 288, no. 1, pp. 298-300, 2005.

[43] R. N. Grass and W. J. Stark, "Gas phase synthesis of fcc-cobalt nanoparticles," Journal of Materials Chemistry, vol. 16, no. 19, pp. 1825-1830, 2006.

[44] T. Jaumann, E. M. M. Ibrahim, S. Hampel, D. Maier, A. Leonhardt, and B. Büchner, "The synthesis of superparamagnetic cobalt nanoparticles encapsulated in carbon through highpressure CVD," Chemical Vapor Deposition, vol. 19, pp. 228-234, 2013.

[45] G. N. Kakazei, Y. G. Pogorelov, A. M. L. Lopes et al., "Tunnel magnetoresistance and magnetic ordering in ion-beam sputtered $\mathrm{Co}_{80} \mathrm{Fe}_{20} / \mathrm{Al}_{2} \mathrm{O}_{3}$ discontinuous multilayers," Journal of Applied Physics, vol. 90, no. 8, pp. 4044-4048, 2001. 
[46] T. Seki, H. Iwama, T. Shima, and K. Takanashi, "Size dependence of the magnetization reversal process in microfabricated $\mathrm{Ll}_{0}$ FePt nano dots," Journal of Physics D, vol. 44, no. 33, Article ID 335001, 2011.

[47] M. Schneider, H. Hoffmann, and J. Zweck, "Lorentz microscopy of circular ferromagnetic permalloy nanodisks," Applied Physics Letters, vol. 77, no. 18, pp. 2909-2911, 2000.

[48] M. Hanson, O. Kazakova, P. Blomqvist, R. Wäppling, and B. Nilsson, "Magnetic domain structures in submicron-size particles of epitaxial $\mathrm{Fe}$ (001) films: shape anisotropy and thickness dependence," Physical Review B, vol. 66, no. 14, Article ID 144419, 2002.

[49] M. Hehn, K. Ounadjela, J.-P. Bucher et al., "Nanoscale magnetic domains in mesoscopic magnets," Science, vol. 272, no. 5269, pp. 1782-1785, 1996.

[50] J. Moritz, L. Buda, B. Dieny et al., "Writing and reading bits on pre-patterned media," Applied Physics Letters, vol. 84, no. 9, pp. 1519-1521, 2004.

[51] T. Shinjo, T. Okuno, R. Hassdorf, K. Shigeto, and T. Ono, "Magnetic vortex core observation in circular dots of permalloy," Science, vol. 289, no. 5481, pp. 930-932, 2000.

[52] D. C. Ralph and M. D. Stiles, "Spin transfer torques," Journal of Magnetism and Magnetic Materials, vol. 320, no. 7, pp.1190-1216, 2008.

[53] S. S. P. Parkin, M. Hayashi, and L. Thomas, "Magnetic domainwall racetrack memory," Science, vol. 320, no. 5873, pp. 190-194, 2008.

[54] N. Kikuchi, S. Okamoto, O. Kitakami, Y. Shimada, and K. Fukamichi, "Sensitive detection of irreversible switching in a single FePt nanosized dot," Applied Physics Letters, vol. 82, no. 24, pp. 4313-4315, 2003.

[55] D. Wang, T. Seki, K. Takanashi, and T. Shima, "Magnetization reversal process in microfabricated $\mathrm{L}_{0}$ - FePt dots," Journal of Physics D, vol. 41, no. 19, Article ID 195008, 2008.

[56] O. Hellwig, L. J. Heyderman, O. Petracic, and H. Zabel, “Competing interactions in patterned and self-assembled magnetic nanostructures," in Springer Tracts in Modern Physics, vol. 246, pp. 189-234, Springer, Berlin, Germany, 2013.

[57] S. A. Claridge, A. W. Castleman Jr., S. N. Khanna, C. B. Murray, A. Sen, and P. S. Weiss, "Cluster-assembled materials," ACS Nano, vol. 3, no. 2, pp. 244-255, 2009.

[58] M. P. Pileni, "Supracrystals of inorganic nanocrystals: an open challenge for new physical properties," Accounts of Chemical Research, vol. 41, no. 12, pp. 1799-1809, 2008.

[59] F. X. Redl, K.-S. Cho, C. B. Murray, and S. O'Brien, “Threedimensional binary superlattices of magnetic nanocrystals and semiconductor quantum dots," Nature, vol. 423, no. 6943, pp. 968-971, 2003.

[60] D. Babonneau, F. Petroff, J.-L. Maurice, F. Fettar, A. Vaurès, and A. Naudon, "Evidence for a self-organized growth in granular $\mathrm{Co} / \mathrm{Al}_{2} \mathrm{O}_{3}$ multilayers," Applied Physics Letters, vol. 76, no. 20, pp. 2892-2894, 2000.

[61] X. Chen, S. Bedanta, O. Petracic et al., "Superparamagnetism versus superspin glass behavior in dilute magnetic nanoparticle systems," Physical Review B, vol. 72, no. 21, Article ID 214436, 2005.

[62] O. Petracic, D. Mishra, D. Greving et al., "Structural and magnetic correlations in iron oxide nanoparticle superlattices," Unpublished.

[63] C. Binns, K. N. Trohidou, J. Bansmann et al., “The behaviour of nanostructured magnetic materials produced by depositing gas-phase nanoparticles," Journal of Physics D, vol. 38, no. 22, pp. R357-R379, 2005.

[64] B. Balasubramanian, R. Skomski, X. Li et al., "Cluster synthesis and direct ordering of rare-earth transition-metal nanomagnets," Nano Letters, vol. 11, no. 4, pp. 1747-1752, 2011.

[65] F. Li, D. P. Josephson, and A. Stein, "Colloidal assembly: the road from particles to colloidal molecules and crystals," Angewandte Chemie (International Edition), vol. 50, no. 2, pp. 360-388, 2011.

[66] K. J. M. Bishop, C. E. Wilmer, S. Soh, and B. A. Grzybowski, "Nanoscale forces and their uses in self-assembly," Small, vol. 5, no. 14, pp. 1600-1630, 2009.

[67] F. Marlow, M. Muldarisnur, P. Sharifi, R. Brinkmann, and C. Mendive, "Opals: status and prospects," Angewandte Chemie (International Edition), vol. 48, no. 34, pp. 6212-6233, 2009.

[68] S. Disch, E. Wetterskog, R. P. Hermann et al., "Shape induced symmetry in self-assembled mesocrystals of iron oxide nanocubes," Nano Letters, vol. 11, no. 4, pp. 1651-1656, 2011.

[69] D. Mishra, D. Greving, G. A. Badini Confalonieri et al., "Growth modes of nanoparticle superlattice thin films," Unpublished.

[70] A. Dong, J. Chen, P. M. Vora, J. M. Kikkawa, and C. B. Murray, "Binary nanocrystal superlattice membranes self-assembled at the liquid-air interface," Nature, vol. 466, no. 7305, pp. 474-477, 2010.

[71] J. Chen, X. Ye, and C. B. Murray, "Systematic electron crystallographic studies of self-assembled binary nanocrystal superlattices," ACS Nano, vol. 4, no. 4, pp. 2374-2381, 2010.

[72] M. J. Benitez, D. Mishra, P. Szary et al., "Structural and magnetic characterization of self-assembled iron oxide nanoparticle arrays," Journal of Physics Condensed Matter, vol. 23, no. 12, Article ID 126003, 2011.

[73] J. W. Cheon, J. I. Park, J. S. Choi et al., "Magnetic superlattices and their nanoscale phase transition effects", Proceedings of the National Academy of Sciences of the United States of America, vol. 103, no. 9, pp. 3023-3027, 2006.

[74] D. Parker, I. Lisiecki, and M. P. Pileni, "Structural and magnetic characterization of self-assembled iron oxide nanoparticle arrays," Journal of Physical Chemistry Letters, vol. 23, no. 12, Article ID 126003, 2011.

[75] W. Kleemann, O. Petracic, C. Binek et al., "Interacting ferromagnetic nanoparticles in discontinuous $\mathrm{Co}_{80} \mathrm{Fe}_{20} / \mathrm{Al}_{2} \mathrm{O}_{3}$ multilayers: from superspin glass to reentrant superferromagnetism," Physical Review B, vol. 63, no. 13, Article ID 134423, 2001.

[76] O. Petracic, X. Chen, S. Bedanta et al., "Collective states of interacting ferromagnetic nanoparticles," Journal of Magnetism and Magnetic Materials, vol. 300, no. 1, pp. 192-197, 2006.

[77] S. Bedanta and W. Kleemann, "Supermagnetism," Journal of Physics D, vol. 42, no. 1, Article ID 013001, 2009.

[78] L. Néel, “Théorie du trainage magnétique des ferromagnétiques en grains fins avec applications aux terres cuites," Annales Geophysique, vol. 5, pp. 99-136, 1949.

[79] L. Weil, L. Gruner, and A. Deschamps, "Orientation des précipitations du cobalt dans un alliage CuCo," Comptes Rendus, vol. 244, p. 2143, 1957.

[80] A. Knappwost, "Kollektivparamagnetismus und volumen magnetisierter aerosole," Zeitschrift für Elektrochemie, vol. 61, pp. 1328-1334, 1957.

[81] R. Hahn and E. Kneller, Zeitschrift für Metallkunde, vol. 49, pp. 426-441, 1958.

[82] A. E. Berkowitz and P. J. Flanders, "Precipitation in a beta-brassfe alloy," Journal of Applied Physics, vol. 30, Article ID S111, 1959. 
[83] W. F. Brown, "Thermal fluctuations of a single-domain particle," Physical Review, vol. 130, no. 5, pp. 1677-1686, 1963.

[84] W. F. Brown Jr., "Relaxational behavior of fine magnetic particles," Journal of Applied Physics, vol. 30, Article ID S130, 1959.

[85] K. Binder and A. P. Young, "Spin glasses: experimental facts, theoretical concepts, and open questions," Reviews of Modern Physics, vol. 58, no. 4, pp. 801-976, 1986.

[86] G. Parisi, "Order parameter for spin-glasses," Physical Review Letters, vol. 50, no. 24, pp. 1946-1948, 1983.

[87] J. Mydosh, Spin Glasses: An Experimental Introduction, Taylor \& Francis, London, UK, 1993.

[88] C. Djurberg, P. Svedlindh, P. Nordblad, M. F. Hansen, F. Bødker, and S. Mørup, "Dynamics of an interacting particle system: evidence of critical slowing down," Physical Review Letters, vol. 79, no. 25, pp. 5154-5157, 1997.

[89] K. Binder and J. D. Reger, "Theory of orientational glasses: models, concepts, simulations," Advances in Physics, vol. 41, no. 6, pp. 547-627, 1992.

[90] R. Skomski, “Are there superspin glasses?" Journal of Applied Physics, vol. 109, no. 7, Article ID 07E149, 2011.

[91] S. Sahoo, O. Petracic, W. Kleemann et al., "Cooperative versus superparamagnetic behavior of dense magnetic nanoparticles in $\mathrm{Co}_{80} \mathrm{Fe}_{20} / \mathrm{Al}_{2} \mathrm{O}_{3}$ multilayers," Applied Physics Letters, vol. 82, no. 23, pp. 4116-4118, 2003.

[92] R. H. Kodama, A. E. Berkowitz, E. J. McNiff Jr., and S. Foner, "Surface spin disorder in $\mathrm{NiFe}_{2} \mathrm{O}_{4}$ nanoparticles," Physical Review Letters, vol. 77, no. 2, pp. 394-397, 1996.

[93] D. Fiorani, Ed., Surface Effects in Magnetic Nanoparticles, Springer, New York, NY, USA, 2005.

[94] K. Nadeem, H. Krenn, T. Traussing, and I. Letofsky-Papst, "Distinguishing magnetic blocking and surface spin-glass freezing in nickel ferrite nanoparticl," Journal of Applied Physics, vol. 109, no. 1, Article ID 013912, 2011.

[95] E. Winkler, R. D. Zysler, M. Vasquez Mansilla et al., "Surface spin-glass freezing in interacting core-shell $\mathrm{NiO}$ nanoparticles," Nanotechnology, vol. 19, no. 18, Article ID 185702, 2008.

[96] K. L. Lopez Maldonado, P. de la Presa, E. Flores Taviz et al., "Magnetic susceptibility studies of the spin-glass and Verwey transitions in magnetite nanoparticles," Journal of Applied Physics, vol. 113, Article ID 17E132, 2013.

[97] O. Bostanjoglo and K. Roehkel, "Superferromagnetism in gadolinium films," Physica Status Solidi A, vol. 11, no. 1, pp. 161166, 1972.

[98] S. Mørup, M. Bo Madsen, J. Franck, J. Villadsen, and C. J. W. Koch, "A new interpretation of Mössbauer spectra of microcrystalline goethite: "super-ferromagnetism" or "superspin-glass" behaviour?" Journal of Magnetism and Magnetic Materials, vol. 40, no. 1-2, pp. 163-174, 1983.

[99] D. G. Rancourt and J. M. Daniels, "Influence of unequal magnetization direction probabilities on the Mössbauer spectra of superparamagnetic particles," Physical Review B, vol. 29, no. 5, pp. 2410-2414, 1984.

[100] S. Sankar, A. E. Berkowitz, D. Dender et al., "Magnetic correlations in non-percolated $\mathrm{Co}-\mathrm{SiO}_{2}$ granular films," Journal of Magnetism and Magnetic Materials, vol. 221, no. 1-2, pp. 1-9, 2000.

[101] S. Bedanta, J. Rhensius, W. Kleemann, P. Parashar, S. Cardoso, and P. P. Freitas, "Dynamic magnetization properties of a superferromagnetic metal-insulator multilayer observed by magneto-optic Kerr microscopy," Journal of Applied Physics, vol. 105, no. 7, Article ID 07C306, 2009.
[102] X. Chen, O. Sichelschmidt, W. Kleemann et al., "Domain wall relaxation, creep, sliding, and switching in superferromagnetic discontinuous $\mathrm{Co}_{80} \mathrm{Fe}_{20} / \mathrm{Al}_{2} \mathrm{O}_{3}$ multilayers," Physical Review Letters, vol. 89, no. 13, Article ID 137023, 2002.

[103] S. Bedanta, O. Petracic, E. Kentzinger et al., "Superferromagnetic domain state of a discontinuous metal insulator multilayer," Physical Review B, vol. 72, no. 2, Article ID 024419, 2005.

[104] X. Chen, W. Kleemann, O. Petracic, O. Sichelschmidt, S. Cardoso, and P. P. Freitas, "Relaxation and aging of a superferromagnetic domain state," Physical Review B, vol. 68, no. 5, Article ID 054433, 2003.

[105] S. Bedanta, T. Eimüller, W. Kleemann et al., "Overcoming the dipolar disorder in dense CoFe nanoparticle ensembles: superferromagnetism," Physical Review Letters, vol. 98, no. 17, Article ID 176601, 2007.

[106] J. Dorman and D. Fiorani, Eds., Magnetic Properties of Fine Particles, North-Holland, Amsterdam, The Netherlands, 1991.

[107] L. A. Harris, J. D. Goff, A. Y. Carmichael et al., "Magnetite nanoparticle dispersions stabilized with triblock copolymers," Chemistry of Materials, vol. 15, no. 6, pp. 1367-1377, 2003.

[108] A. F. Thünemann, D. Schütt, L. Kaufner, U. Pison, and H. Möhwald, "Maghemite nanoparticles protectively coated with poly(ethylene imine) and poly(ethylene oxide)-blockpoly(glutamic acid)," Langmuir, vol. 22, no. 5, pp. 2351-2357, 2006.

[109] V. Skumryev, S. Stoyanov, Y. Zhang, G. Hadjipanayis, D. Givord, and J. Nogués, "Beating the superparamagnetic limit with exchange bias," Nature, vol. 423, no. 6942, pp. 850-853, 2003.

[110] Ò. Iglesias, A. Labarta, and X. Batlle, "Exchange bias phenomenology and models of core/shell nanoparticles," Journal of Nanoscience and Nanotechnology, vol. 8, no. 6, pp. 2761-2780, 2008.

[111] A. Punnoose, H. Magnone, M. S. Seehra, and J. Bonevich, "Bulk to nanoscale magnetism and exchange bias in $\mathrm{CuO}$ nanoparticles," Physical Review B, vol. 64, no. 17, Article ID 174420, 2001.

[112] R. D. Zysler, E. Winkler, M. Vasquez Mansilla, and D. Fiorani, "Surface effect in the magnetic order of antiferromagnetic nanoparticles," Physica B, vol. 384, no. 1-2, pp. 277-281, 2006.

[113] J. Nogués, V. Skumryev, J. Sort, S. Stoyanov, and D. Givord, "Shell-driven magnetic stability in core-shell nanoparticles," Physical Review Letters, vol. 97, no. 15, Article ID 157203, 2006.

[114] B. Jeyadevan, C. N. Chinnasamy, O. Perales-Perez et al., "Synthesis and magnetic properties of core-shell structured (NiCo)O(AFM)-NiCo(FM) magnetic nanoparticles," IEEE Transactions on Magnetics, vol. 38, no. 5, pp. 2595-2597, 2002.

[115] J. van Lierop, M. A. Schofield, L. H. Lewis, and R. J. Gambino, "Exchange bias in a thin film dispersion of $\mathrm{MnO}$ nanocrystallites in Co," Journal of Magnetism and Magnetic Materials, vol. 264, no. 2-3, pp. 146-152, 2003.

[116] C. Luna, M. del Puerto Morales, C. J. Serna, and M. Vázquez, "Exchange anisotropy in $\mathrm{Co}_{80} \mathrm{Ni}_{20}$ /oxide nanoparticles," Nanotechnology, vol. 15, no. 4, pp. S293-S297, 2004.

[117] A. Tomou, D. Gournis, I. Panagiotopoulos, Y. Huang, G. C. Hadjipanayis, and B. J. Kooi, "Weak ferromagnetism and exchange biasing in cobalt oxide nanoparticle systems," Journal of Applied Physics, vol. 99, no. 12, Article ID 123915, 2006.

[118] Ò. Iglesias, A. Labarta, and X. Batlle, "Exchange bias phenomenology and models of core/shell nanoparticles," Journal of Nanoscience and Nanotechnology, vol. 8, no. 6, pp. 2761-2780, 2008. 
[119] G. Salazar-Alvarez, J. Sort, S. Suriñach, M. D. Baró, and J. Nogués, "Synthesis and size-dependent exchange bias in inverted core-shell $\mathrm{MnO}-\mathrm{Mn3O} 4$ nanoparticles," Journal of the American Chemical Society, vol. 129, no. 29, pp. 9102-9108, 2007.

[120] E. L. Salabaş, A. Rumplecker, F. Kleitz, F. Radu, and F. Schüth, "Exchange anisotropy in nanostructured porous $\mathrm{Co}_{3} \mathrm{O}_{4}$," Nano Letters, vol. 6, pp. 2977-2981, 2006.

[121] J. Nogués, J. Sort, V. Langlais et al., "Exchange bias in nanostructures," Physics Reports, vol. 422, no. 3, pp. 65-117, 2005.

[122] A. Moser, K. Takano, D. T. Margulies et al., "Magnetic recording: advancing into the future," Journal of Physics D, vol. 35, no. 19, pp. R157-R167, 2002.

[123] W. Andrä, U. Häfeli, R. Hergt, and R. Misri, "Application of magnetic particles in medicine and biology," in Handbook of Magnetism and Advanced Magnetic Materials, Vol. 4: Novel Materials, H. Kronmüller and S. P. S. Parkin, Eds., pp. 253622568, John Wiley \& Sons, Chichester, UK, 2007.

[124] Y. Shiroishi, K. Fukuda, I. Tagawa et al., "Future options for HDD storage," IEEE Transactions on Magnetics, vol. 45, no. 10, pp. 3816-3822, 2009.

[125] B. D. Terris and T. Thomson, "Nanofabricated and selfassembled magnetic structures as data storage media," Journal of Physics D, vol. 38, no. 12, pp. R199-R222, 2005.

[126] Y. Guo, P. Wang, M.-M. Chen et al., "MRAM array with coupled soft-adjacent magnetic layer," Journal of Applied Physics, vol. 97, no. 10, Article ID 10P506, 2005.

[127] D. Weller and A. Moser, "Thermal effect limits in ultrahighdensity magnetic recording," IEEE Transactions on Magnetics, vol. 35, no. 6, pp. 4423-4439, 1999.

[128] O. A. Ivanov, L. V. Solina, V. A. Demshina, and L. M. Magat, "Determination of the anisotropy constant and saturation magnetization and magnetic properties of an iron-platinum alloy," Physics of Metals and Metallography, vol. 35, no. 1, pp. 8185, 1973.

[129] J. Fidler and T. Schrefl, "Micromagnetic modelling-the current state of the art," Journal of Physics D, vol. 33, no. 15, pp. R135R156, 2000.

[130] D. P. Landau and K. Binder, A Guide to Monte Carlo Simulations in Statistical Physics, Cambridge University Press, Cambridge, UK, 2005.

[131] T. L. Gilbert, "A lagrangian formulation of the gyromagnetic equation of the magnetic field," Physical Review, vol. 100, Article ID 1243, 1955.

[132] J.-O. Andersson, C. Djurberg, T. Jonsson, P. Svedlindh, and P. Nordblad, "Monte Carlo studies of the dynamics of an interacting monodispersive magnetic-particle system," Physical Review B, vol. 56, no. 21, pp. 13983-13988, 1997.

[133] Ò. Iglesias and A. Labarta, "Finite-size and surface effects in maghemite nanoparticles: Monte Carlo simulations," Physical Review B, vol. 63, no. 18, Article ID 184416, 2001.

[134] S. Tamaru, J. A. Bain, R. J. M. van de Veerdonk, T. M. Crawford, M. Covington, and M. H. Kryder, "Imaging of quantized magnetostatic modes using spatially resolved ferromagnetic resonance," Journal of Applied Physics, vol. 91, no. 10, p. 8034, 2002.

[135] G. Gubbiotti, G. Carlotti, M. Madami, S. Tacchi, P. Vavassori, and G. Socino, "Setup of a new Brillouin light scattering apparatus with submicrometric lateral resolution and its application to the study of spin modes in nanomagnets," Journal of Applied Physics, vol. 105, no. 7, Article ID 07D521, 2009.
[136] I. N. Krivorotov, N. C. Emley, J. C. Sankey, S. I. Kiselev, D. C. Ralph, and R. A. Buhrman, "Time-domain measurements of nanomagnet dynamics driven by spin-transfer torques," Science, vol. 307, no. 5707, pp. 228-231, 2005.

[137] Y. Acremann, J. P. Strachan, V. Chembrolu et al., “Time-resolved imaging of spin transfer switching: beyond the macrospin concept," Physical Review Letters, vol. 96, no. 21, Article ID 217202, 2006.

[138] T. J. Silva, C. S. Lee, T. M. Crawford, and C. T. Rogers, "Inductive measurement of ultrafast magnetization dynamics in thin-film Permalloy," Journal of Applied Physics, vol. 85, no. 11, pp. 78497862, 1999.

[139] C. Herring and C. Kittel, "On the theory of spin waves in ferromagnetic media," Physical Review, vol. 81, no. 5, pp. 869880, 1951.

[140] R. W. Damon and J. R. Eshbach, "Magnetostatic modes of a ferromagnet slab," Journal of Physics and Chemistry of Solids, vol. 19, pp. 308-320, 1961.

[141] R.-P. Pan, H. D. Wei, and Y. R. Shen, "Optical second-harmonic generation from magnetized surfaces," Physical Review B, vol. 39, no. 2, pp. 1229-1234, 1989.

[142] P. Kasiraj, R. M. Shelby, J. S. Best, and D. E. Horne, "Magnetic domain imaging with a scanning Kerr effect microscope," IEEE Transactions on Magnetics, vol. 22, no. 5, pp. 837-839, 1986.

[143] M. R. Freeman, R. R. Ruf, and R. J. Gambino, "Picosecond pulsed magnetic fields for studies of ultrafast magnetic phenomena," IEEE Transactions on Magnetics, vol. 27, no. 6, pp. 4840-4842, 1991.

[144] D. H. Auston, "Picosecond optoelectronic switching and gating in silicon," Applied Physics Letters, vol. 26, no. 3, pp. 101-103, 1975.

[145] M. R. Freeman, M. J. Brady, and J. Smyth, "Extremely high frequency pulse magnetic resonance by picosecond magnetooptic sampling," Applied Physics Letters, vol. 60, no. 20, pp. 2555-2557, 1992.

[146] A. Y. Elezzabi, M. R. Freeman, and M. Johnson, "Direct measurement of the conduction electron spin-lattice relaxation time T1 in gold," Physical Review Letters, vol. 77, no. 15, pp. 3220 3223, 1996

[147] E. Beaurepaire, J.-C. Merle, A. Daunois, and J.-Y. Bigot, "Ultrafast spin dynamics in ferromagnetic nickel," Physical Review Letters, vol. 76, no. 22, pp. 4250-4253, 1996.

[148] W. K. Hiebert, A. Stankiewicz, and M. R. Freeman, "Direct observation of magnetic relaxation in a small permalloy disk by time-resolved scanning kerr microscopy," Physical Review Letters, vol. 79, no. 6, pp. 1134-1137, 1997.

[149] B. Koopmans, M. van Kampen, J. T. Kohlhepp, and W. J. M. de Jonge, "Ultrafast magneto-optics in nickel: magnetism or optics?" Physical Review Letters, vol. 85, no. 4, pp. 844-847, 2000.

[150] M. van Kampen, C. Jozsa, J. T. Kohlhepp et al., "All-optical probe of coherent spin waves," Physical Review Letters, vol. 88, no. 22, Article ID 227201, 2002.

[151] S. Jung, B. Watkins, L. DeLong, J. B. Ketterson, and V. Chandrasekhar, "Ferromagnetic resonance in periodic particle arrays," Physical Review B, vol. 66, no. 13, Article ID 132401, 2002.

[152] G. Gubbiotti, G. Carlotti, T. Okuno, T. Shinjo, F. Nizzoli, and R. Zivieri, "Brillouin light scattering investigation of dynamic spin modes confined in cylindrical Permalloy dots," Physical Review B, vol. 68, no. 18, Article ID 184409, 2003. 
[153] V. V. Kruglyak, A. Barman, R. J. Hicken, J. R. Childress, and J. A. Katine, "Picosecond magnetization dynamics in nanomagnets: crossover to nonuniform precession," Physical Review B, vol. 71, no. 22, Article ID 220409, 2005.

[154] A. Barman, S. Wang, J. D. Maas et al., "Magneto-optical observation of picosecond dynamics of single nanomagnets," Nano Letters, vol. 6, no. 12, pp. 2939-2944, 2006.

[155] A. Barman, S. Wang, J. Maas et al., "Size dependent damping in picosecond dynamics of single nanomagnets," Applied Physics Letters, vol. 90, no. 20, Article ID 202504, 2007.

[156] V. V. Kruglyak, P. S. Keatley, R. J. Hicken, J. R. Childress, and J. A. Katine, "Dynamic configurational anisotropy in nanomagnets," Physical Review B, vol. 75, no. 2, Article ID 024407, 2007.

[157] A. Laraoui, J. Vénuat, V. Halté, M. Albrecht, E. Beaurepaire, and J.-Y. Bigot, "Study of individual ferromagnetic disks with femtosecond optical pulses," Journal of Applied Physics, vol. 101, no. 9, Article ID 09C105, 2007.

[158] Z. Liu, R. D. Sydora, and M. R. Freeman, "Shape effects on magnetization state transitions in individual 160-nm diameter Permalloy disks," Physical Review B, vol. 77, no. 17, Article ID 174410, 2008.

[159] P. S. Keatley, P. Gangmei, M. Dvornik, R. J. Hicken, J. R. Childress, and J. A. Katine, "Large amplitude magnetization dynamics and the suppression of edge modes in a single nanomagnet," Applied Physics Letters, vol. 98, no. 8, Article ID 082506, 2011.

[160] V. V. Kruglyak, P. S. Keatley, A. Neudert, R. J. Hicken, J. R. Childress, and J. A. Katine, "Imaging collective magnonic modes in 2D arrays of magnetic nanoelements," Physical Review Letters, vol. 104, no. 2, Article ID 027201, 2010.

[161] B. Rana, S. Pal, S. Barman, Y. Fukuma, Y. Otani, and A. Barman, "All-optical excitation and detection of picosecond dynamics of ordered arrays of nanomagnets with varying areal density," Applied Physics Express, vol. 4, no. 11, Article ID 113003, 2011.

[162] B. Rana, D. Kumar, S. Barman et al., "Anisotropy in collective precessional dynamics in arrays of $\mathrm{Ni}_{80} \mathrm{Fe}_{20}$ nanoelements," Journal of Applied Physics, vol. 111, Article ID 07D503, 2012.

[163] B. Rana, D. Kumar, S. Barman et al., "Detection of picosecond magnetization dynamics of $50 \mathrm{~nm}$ magnetic dots down to the single dot regime," ACS Nano, vol. 5, no. 12, pp. 9559-9565, 2011.

[164] A. Barman and S. Barman, "Dynamic dephasing of magnetization precession in arrays of thin magnetic elements," Physical Review B, vol. 79, no. 14, Article ID 144415, 2009.

[165] S. Saha, R. Mandal, S. Barman et al., “Tunable magnonic spectra in two-dimensional magnonic crystals with variable lattice symmetry," Advanced Functional Materials, vol. 23, pp. 23782386, 2013.

[166] B. K. Mahato, B. Rana, D. Kumar et al., "Configurational anisotropic spin waves in cross-shaped $\mathrm{Ni}_{80} \mathrm{Fe}_{20}$ nanoelements," Applied Physics Letters, vol. 102, Article ID 192402, 2013.

[167] S. Saha, S. Barman, J. Ding, A. O. Adeyeye, and A. Barman, "Time-domain study of spin-wave dynamics in twodimensional arrays of bi-component magnetic structures," Applied Physics Letters, vol. 102, Article ID 242409, 2013. 

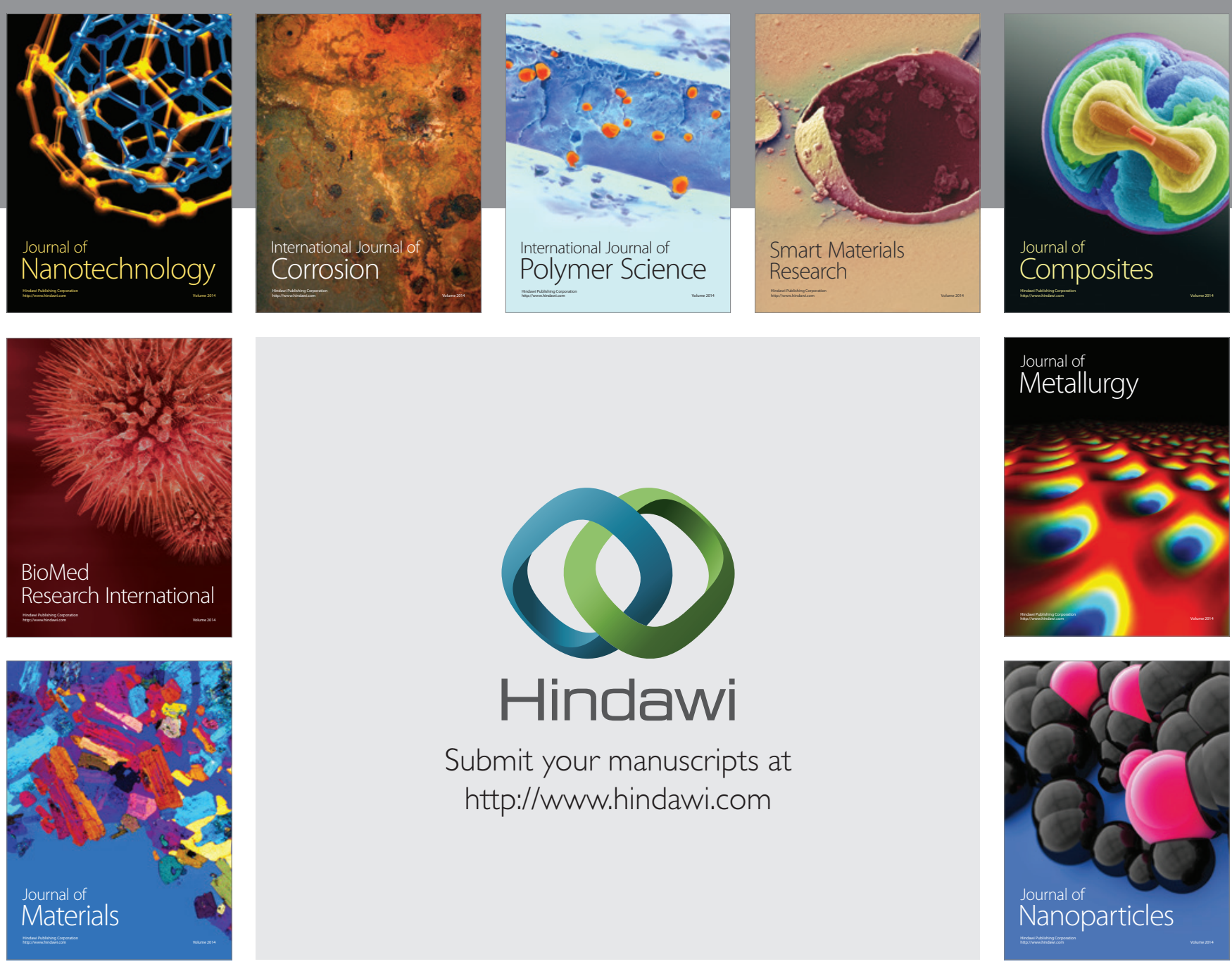

Submit your manuscripts at http://www.hindawi.com
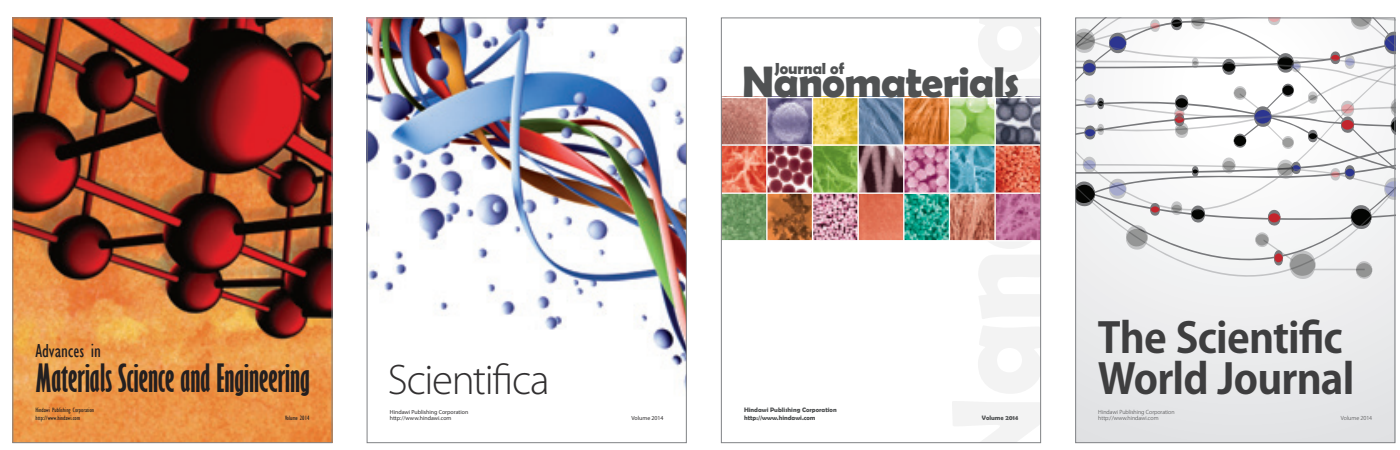

\section{The Scientific World Journal}
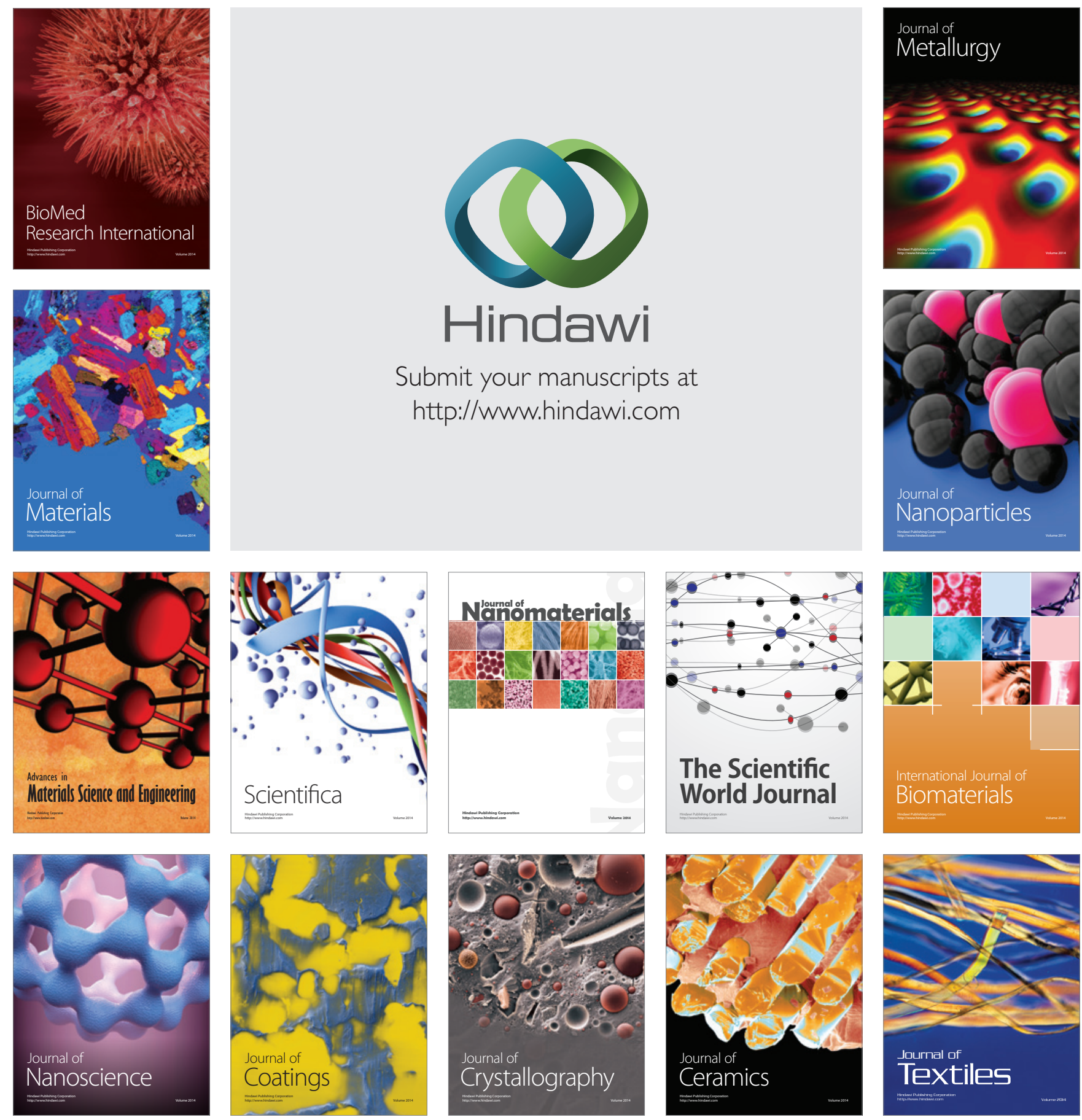